\title{
Cycloneophylpalladium(IV) Complexes: Formation by Oxidative Addition and Selectivity of their Reductive Elimination Reactions
}

\author{
Supporting Information \\ Ava Behnia, Mahmood A. Fard, Johanna M. Blacquiere* and Richard J. Puddephatt* \\ Department of Chemistry \\ University of Western Ontario \\ London, Ontario, Canada, N6A 5B7
}

Table of Contents

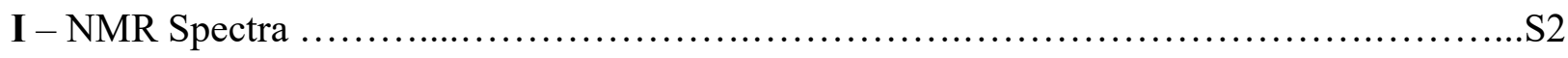

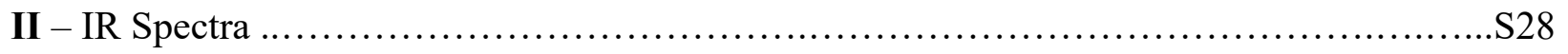

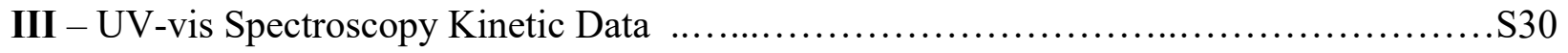

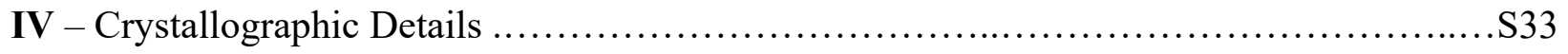

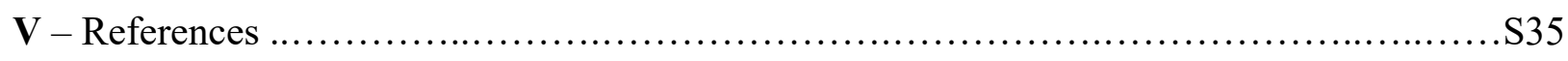




\section{I - NMR Spectra}

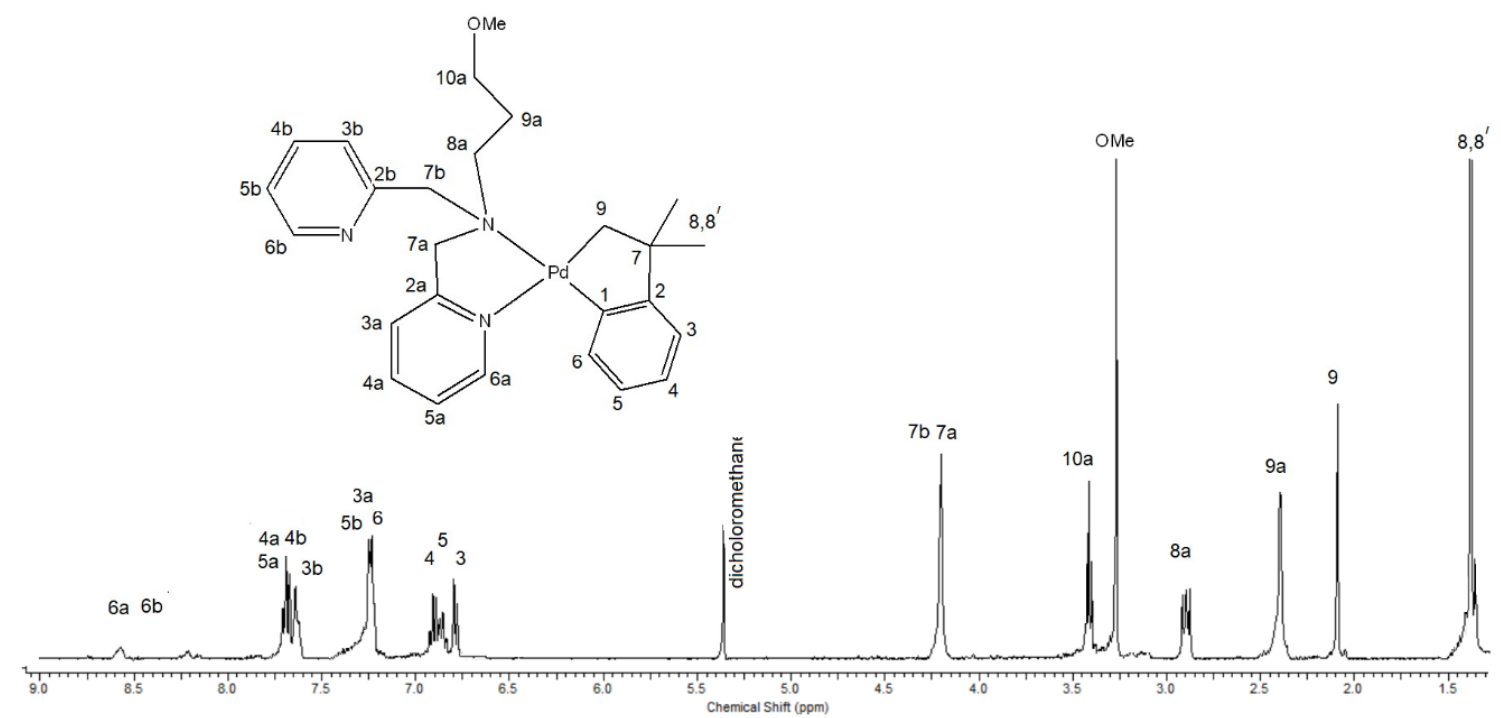

Figure S1. ${ }^{1} \mathrm{H}$ NMR spectrum of 2, $\left[\mathrm{Pd}\left(\mathrm{CH}_{2} \mathrm{CMe}_{2} \mathrm{C}_{6} \mathrm{H}_{4}\right)\left(\kappa^{2}-N, N^{\prime}-\mathbf{L 2}\right)\right]$, at $25{ }^{\circ} \mathrm{C}(600 \mathrm{MHz}$, $\left.\mathrm{CD}_{2} \mathrm{Cl}_{2}\right)$.

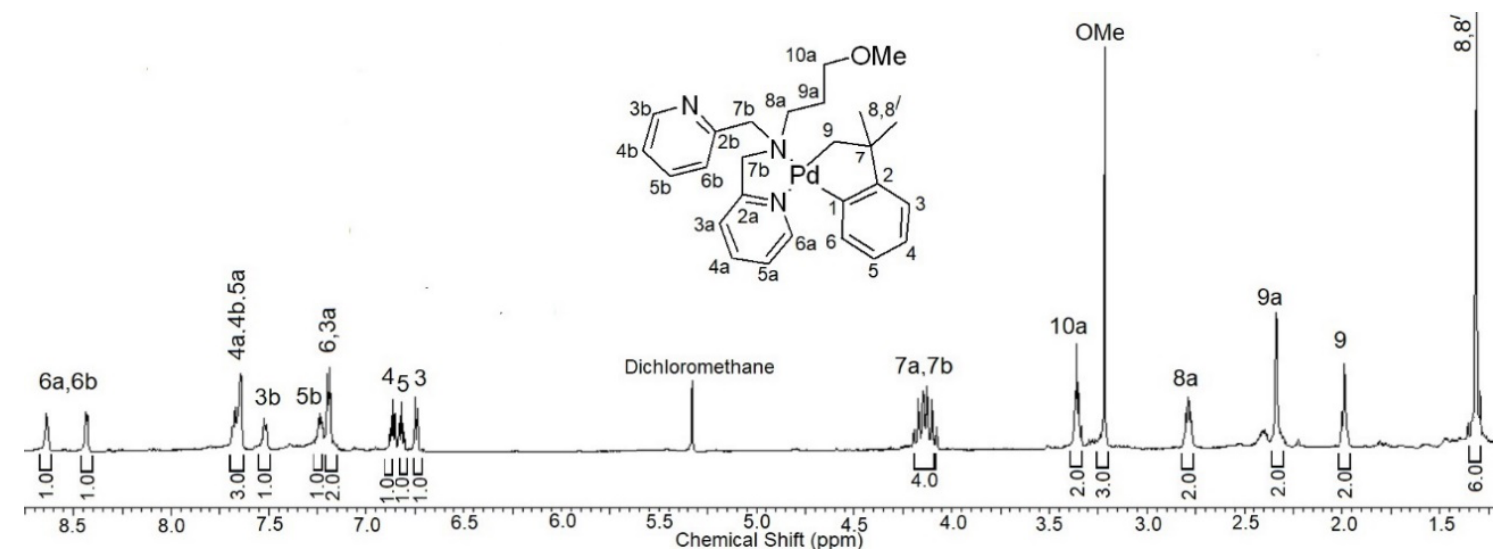

Figure S2. ${ }^{1} \mathrm{H}$ NMR spectrum of 2, $\left[\mathrm{Pd}\left(\mathrm{CH}_{2} \mathrm{CMe}_{2} \mathrm{C}_{6} \mathrm{H}_{4}\right)\left(\kappa^{2}-N, N^{\prime}-\mathbf{L} 2\right)\right]$, at $-30{ }^{\circ} \mathrm{C}(600 \mathrm{MHz}$, $\left.\mathrm{CD}_{2} \mathrm{Cl}_{2}\right)$. 


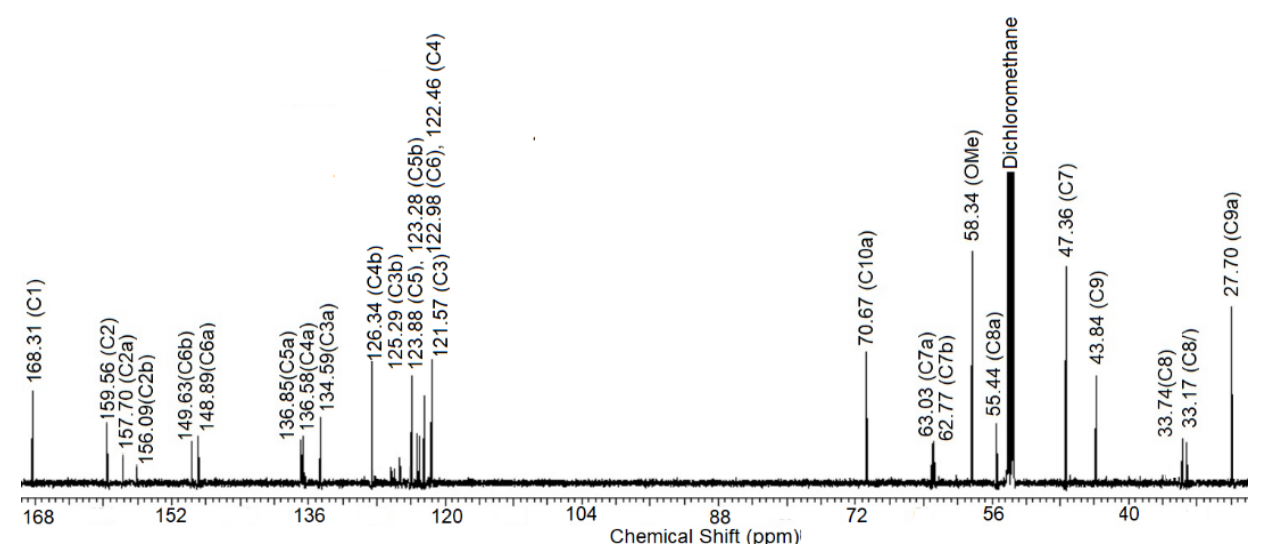

Figure S3. ${ }^{13} \mathrm{C}\left\{{ }^{1} \mathrm{H}\right\}$ NMR spectrum of 2, $\left[\mathrm{Pd}\left(\mathrm{CH}_{2} \mathrm{CMe}_{2} \mathrm{C}_{6} \mathrm{H}_{4}\right)\left(\kappa^{2}-N, N^{\prime}-\mathbf{L 2}\right)\right]$, at $-30{ }^{\circ} \mathrm{C}(151$ $\left.\mathrm{MHz}, \mathrm{CD}_{2} \mathrm{Cl}_{2}\right)$.

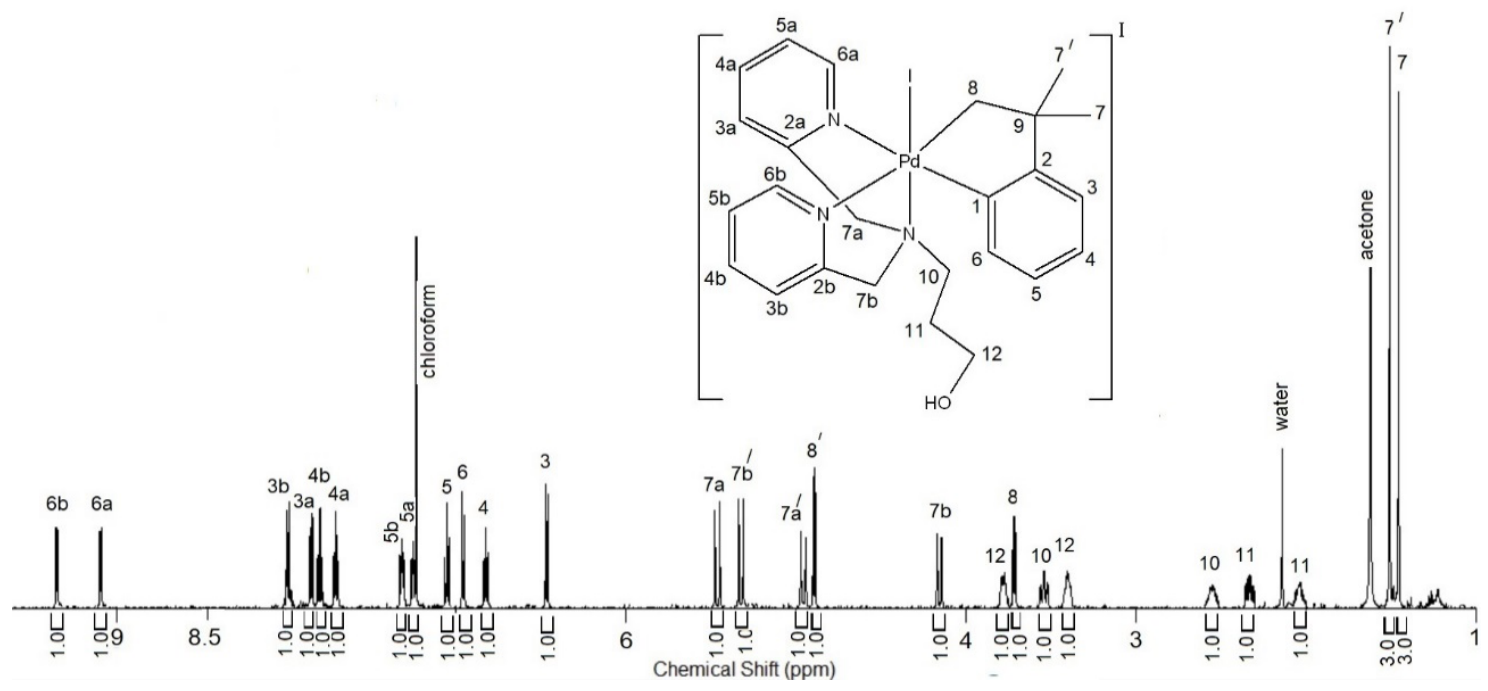

Figure S4. ${ }^{1} \mathrm{H}$ NMR spectrum of $3,\left[\mathrm{PdI}\left(\mathrm{CH}_{2} \mathrm{CMe}_{2} \mathrm{C}_{6} \mathrm{H}_{4}\right)\left(\kappa^{3}-N, N^{\prime}, N^{\prime \prime}-\mathbf{L 1}\right)\right][\mathrm{I}]$, at $25^{\circ} \mathrm{C}(600 \mathrm{MHz}$, $\left.\mathrm{CDCl}_{3}\right)$. 


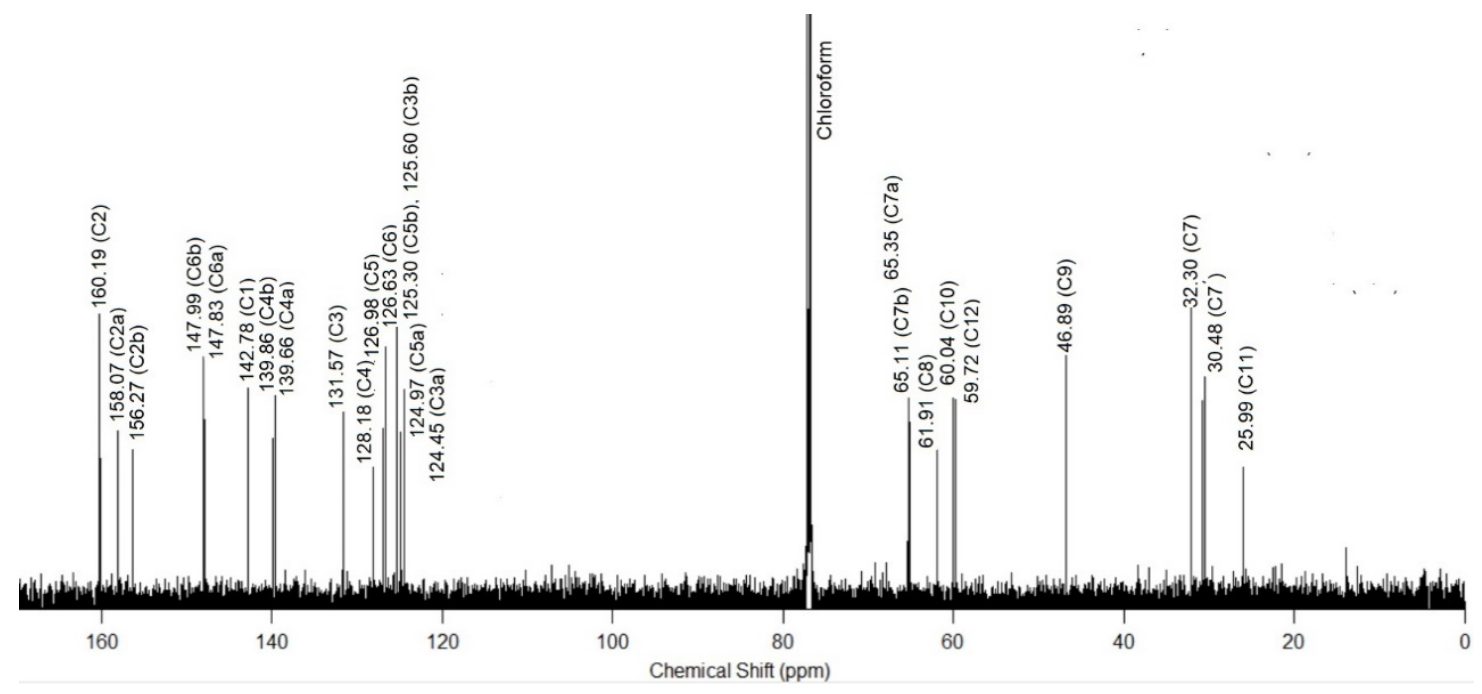

Figure S5. ${ }^{13} \mathrm{C}\left\{{ }^{1} \mathrm{H}\right\}$ NMR spectrum of 3, $\left[\mathrm{PdI}\left(\mathrm{CH}_{2} \mathrm{CMe}_{2} \mathrm{C}_{6} \mathrm{H}_{4}\right)\left(\kappa^{3}-N, N^{\prime}, N^{\prime \prime}-\mathbf{L 1}\right)\right][\mathrm{I}]$, at $25^{\circ} \mathrm{C}(151$ $\left.\mathrm{MHz}, \mathrm{CDCl}_{3}\right)$.

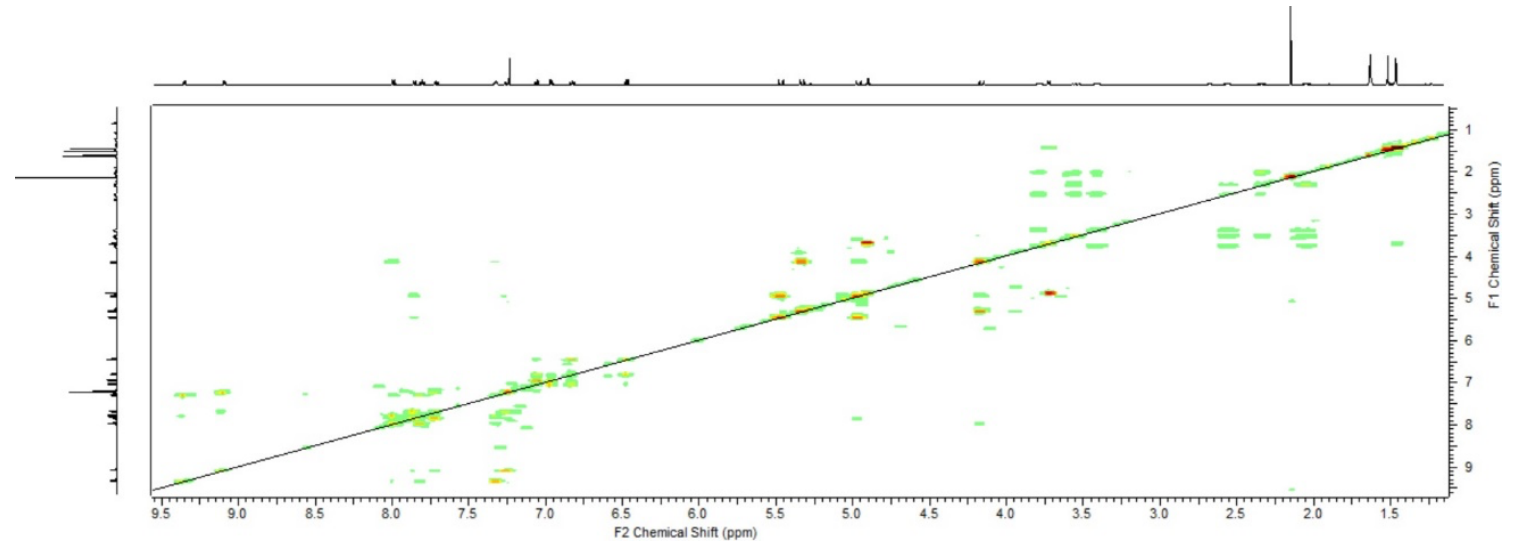

Figure S6. ${ }^{1} \mathrm{H}-{ }^{1} \mathrm{H}$ gCOSY NMR spectrum of 3, $\left[\mathrm{PdI}\left(\mathrm{CH}_{2} \mathrm{CMe}_{2} \mathrm{C}_{6} \mathrm{H}_{4}\right)\left(\kappa^{3}-N, N^{\prime}, N^{\prime \prime}-\mathbf{L} \mathbf{1}\right)\right][\mathrm{I}]$, at $25^{\circ} \mathrm{C}$ (600 $\left.\mathrm{MHz}, \mathrm{CDCl}_{3}\right)$. 


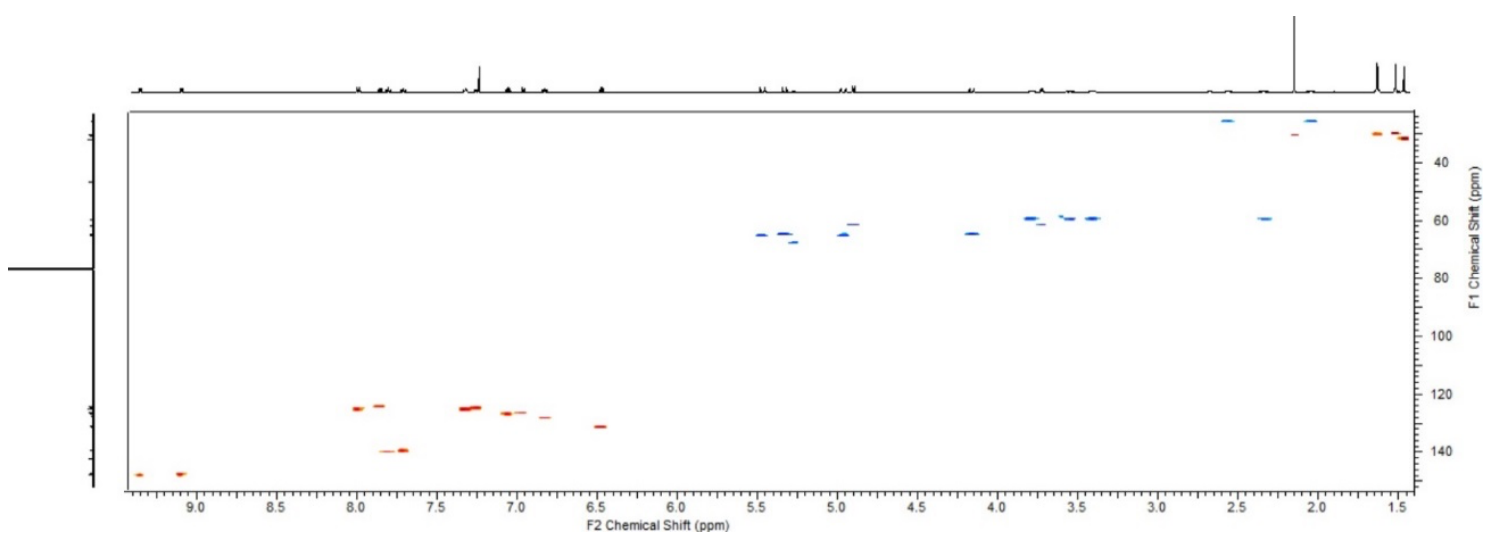

Figure S7. ${ }^{1} \mathrm{H}-{ }^{13} \mathrm{C}$ HSQC NMR spectrum of 3, $\left[\mathrm{PdI}\left(\mathrm{CH}_{2} \mathrm{CMe}_{2} \mathrm{C}_{6} \mathrm{H}_{4}\right)\left(\kappa^{3}-N, N^{\prime}, N^{\prime \prime}-\mathbf{L 1}\right)\right][\mathrm{I}]$, at $25^{\circ} \mathrm{C}$ (600 MHz, $\mathrm{CDCl}_{3}$ ).

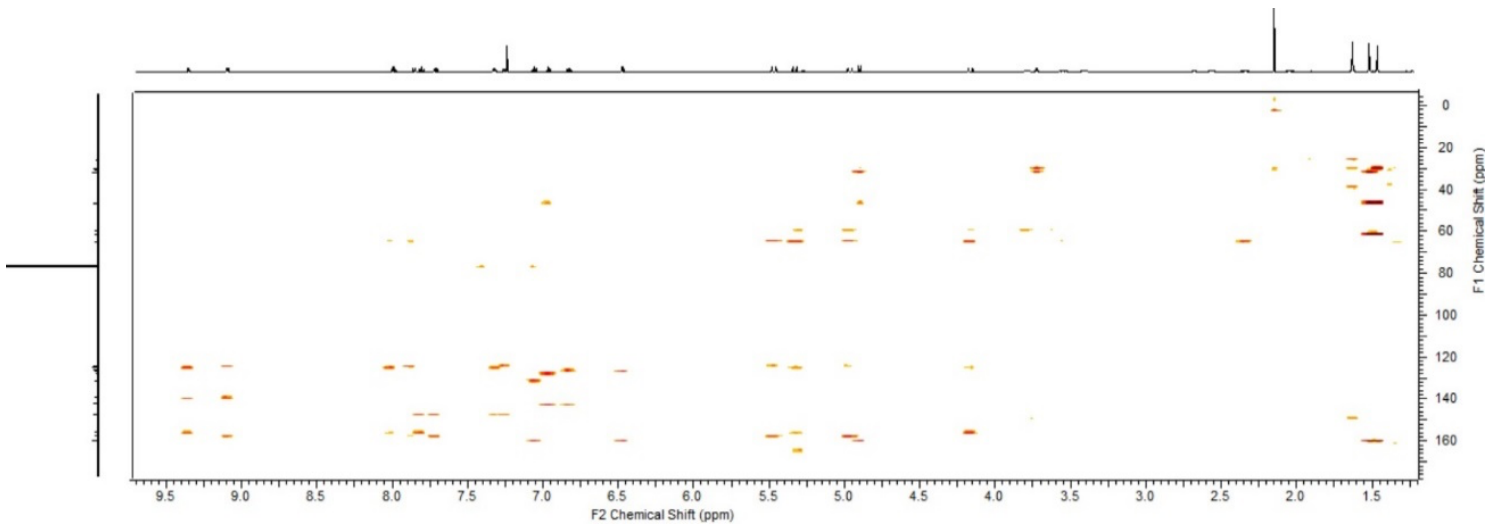

Figure S8. ${ }^{1} \mathrm{H}-{ }^{13} \mathrm{C}$ HMBC NMR spectrum of 3, $\left[\mathrm{PdI}\left(\mathrm{CH}_{2} \mathrm{CMe}_{2} \mathrm{C}_{6} \mathrm{H}_{4}\right)\left(\kappa^{3}-N, N^{\prime}, N^{\prime \prime}-\mathbf{L} \mathbf{1}\right)\right][\mathrm{I}]$, at $25^{\circ} \mathrm{C}$ $\left(600 \mathrm{MHz}, \mathrm{CDCl}_{3}\right)$. 


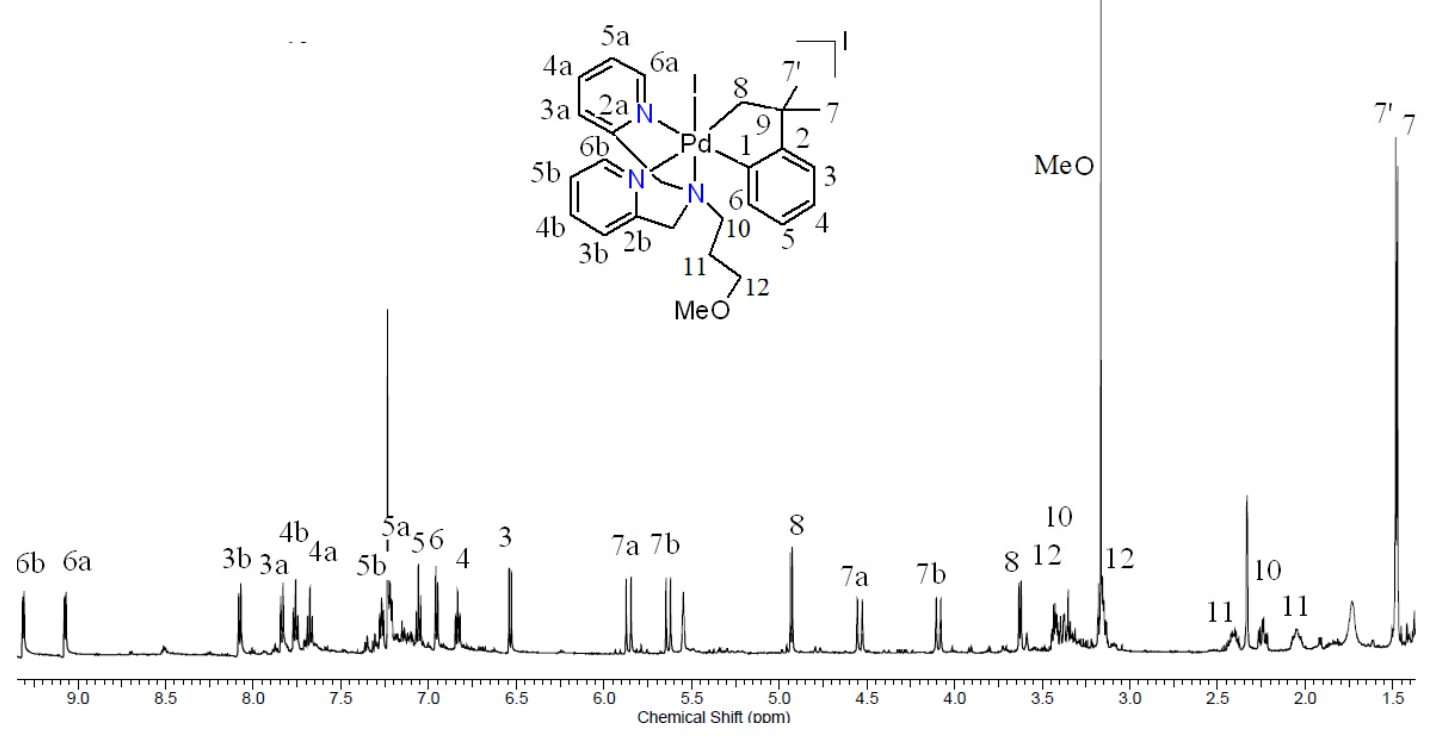

Figure S9. ${ }^{1} \mathrm{H}$ NMR spectrum of 4, $\left[\mathrm{PdI}\left(\mathrm{CH}_{2} \mathrm{CMe}_{2} \mathrm{C}_{6} \mathrm{H}_{4}\right)\left(\kappa^{3}-N, N^{\prime}, N^{\prime \prime}-\mathbf{L 2}\right)\right][\mathrm{I}]$, at $25^{\circ} \mathrm{C}(600 \mathrm{MHz}$, $\left.\mathrm{CDCl}_{3}\right)$.

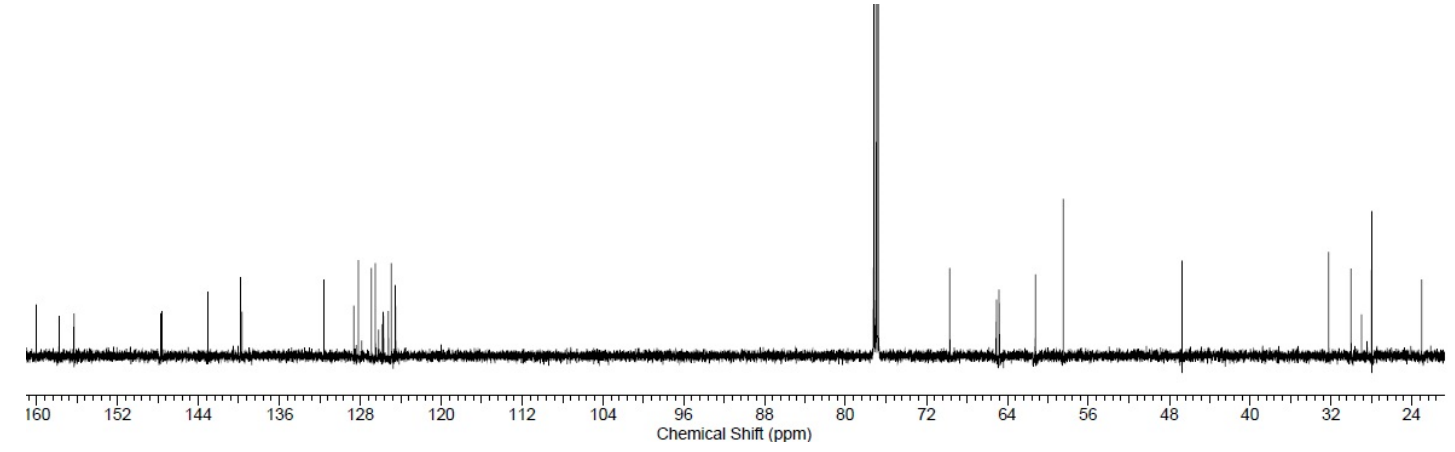

Figure S10. ${ }^{13} \mathrm{C}\left\{{ }^{1} \mathrm{H}\right\}$ NMR spectrum of 4, $\left[\mathrm{PdI}\left(\mathrm{CH}_{2} \mathrm{CMe}_{2} \mathrm{C}_{6} \mathrm{H}_{4}\right)\left(\kappa^{3}-N, N^{\prime}, N^{\prime \prime}-\mathbf{L} 2\right)\right][\mathrm{I}]$, at $25^{\circ} \mathrm{C}(151$ $\mathrm{MHz}, \mathrm{CDCl}_{3}$ ). 


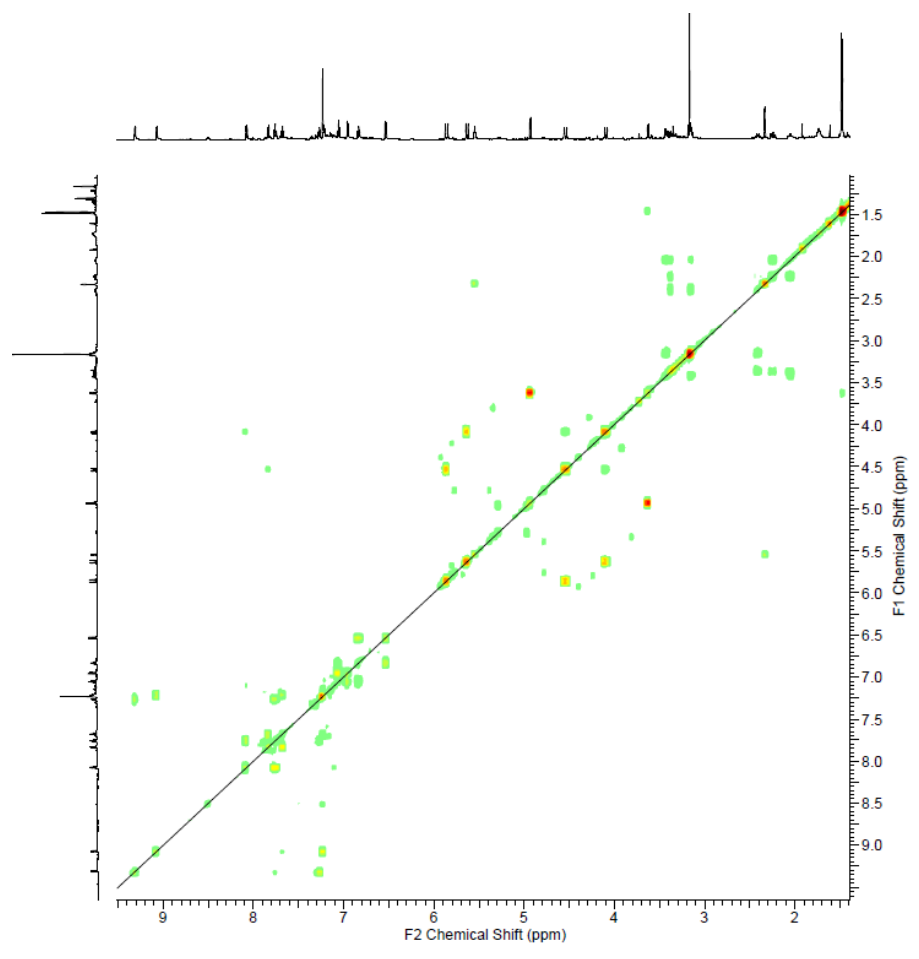

Figure S11. ${ }^{1} \mathrm{H}-{ }^{1} \mathrm{H}$ gCOSY NMR spectrum of 4, $\left[\mathrm{PdI}\left(\mathrm{CH}_{2} \mathrm{CMe}_{2} \mathrm{C}_{6} \mathrm{H}_{4}\right)\left(\kappa^{3}-N, N^{\prime}, N^{\prime \prime}-\mathbf{L} 2\right)\right][\mathrm{I}]$, at $25^{\circ} \mathrm{C}$ $\left(600 \mathrm{MHz}, \mathrm{CDCl}_{3}\right)$.

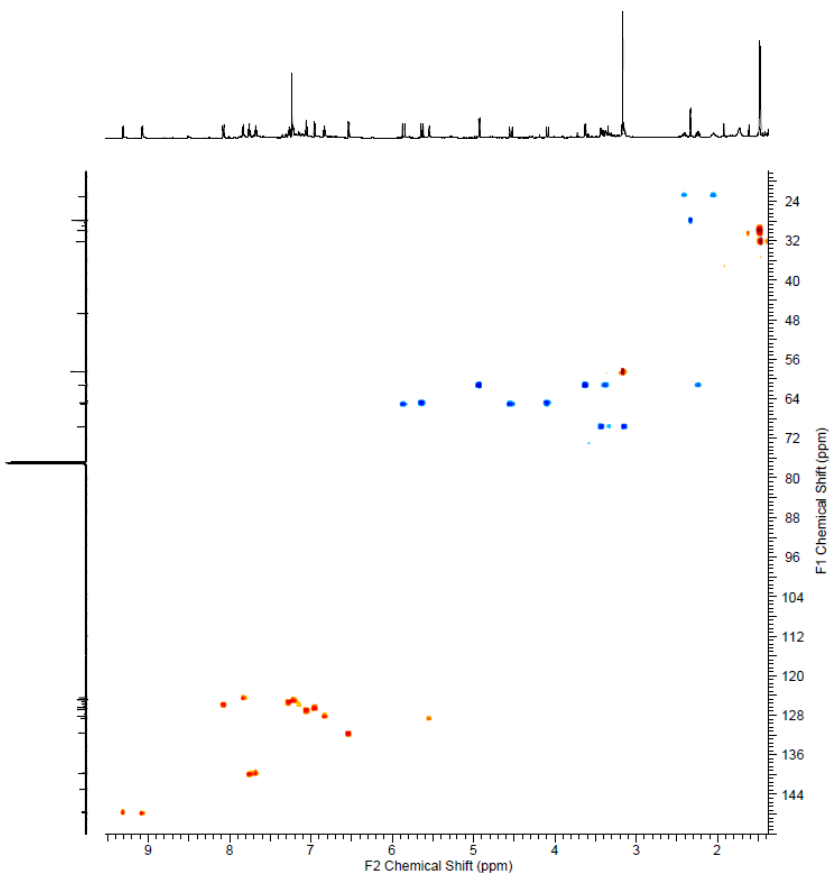

Figure S12. ${ }^{1} \mathrm{H}-{ }^{13} \mathrm{C}$ HSQC NMR spectrum of 4, $\left[\mathrm{PdI}\left(\mathrm{CH}_{2} \mathrm{CMe}_{2} \mathrm{C}_{6} \mathrm{H}_{4}\right)\left(\kappa^{3}-N, N^{\prime}, N^{\prime \prime}-\mathbf{L 2}\right)\right][\mathrm{I}]$, at $25^{\circ} \mathrm{C}$ $\left(600 \mathrm{MHz}, \mathrm{CDCl}_{3}\right)$. 


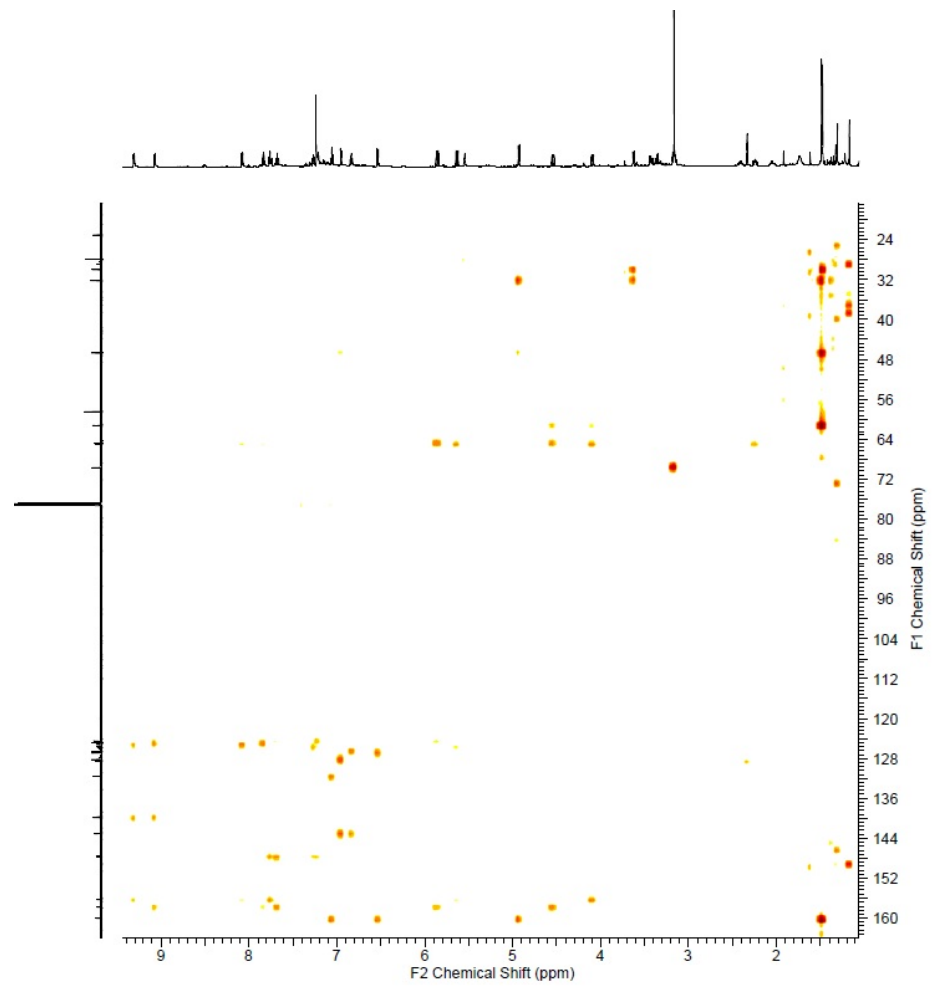

Figure S13. ${ }^{1} \mathrm{H}-{ }^{13} \mathrm{C}$ HMBC NMR spectrum of 4, $\left[\mathrm{PdI}\left(\mathrm{CH}_{2} \mathrm{CMe}_{2} \mathrm{C}_{6} \mathrm{H}_{4}\right)\left(\kappa^{3}-N, N^{\prime}, N^{\prime \prime}-\mathbf{L} 2\right)\right][\mathrm{I}]$, at $25^{\circ} \mathrm{C}\left(600 \mathrm{MHz}, \mathrm{CDCl}_{3}\right)$.

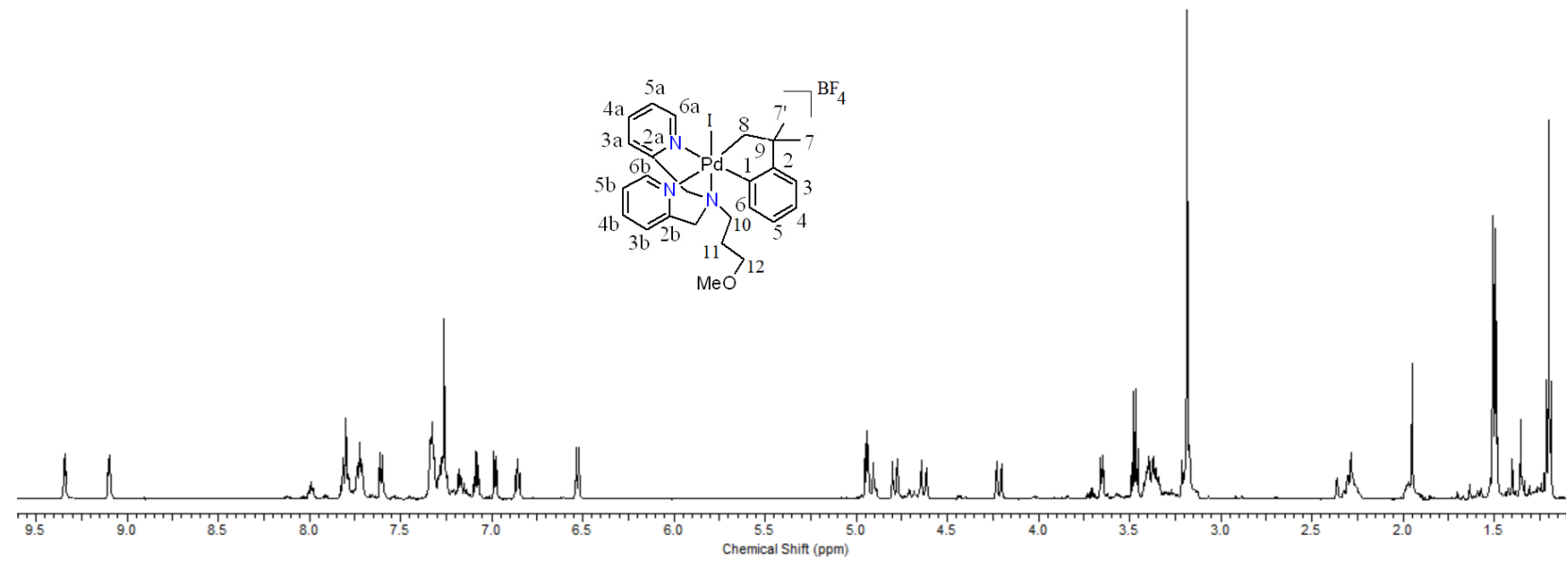

Figure S14. ${ }^{1} \mathrm{H}$ NMR spectrum of $\mathbf{4 a},\left[\mathrm{PdI}\left(\mathrm{CH}_{2} \mathrm{CMe}_{2} \mathrm{C}_{6} \mathrm{H}_{4}\right)\left(\kappa^{3}-N, N^{\prime}, N^{\prime \prime}-\mathbf{L} 2\right)\right]\left[\mathrm{BF}_{4}\right]$, at $25^{\circ} \mathrm{C}(600$ $\left.\mathrm{MHz}, \mathrm{CDCl}_{3}\right)$. 


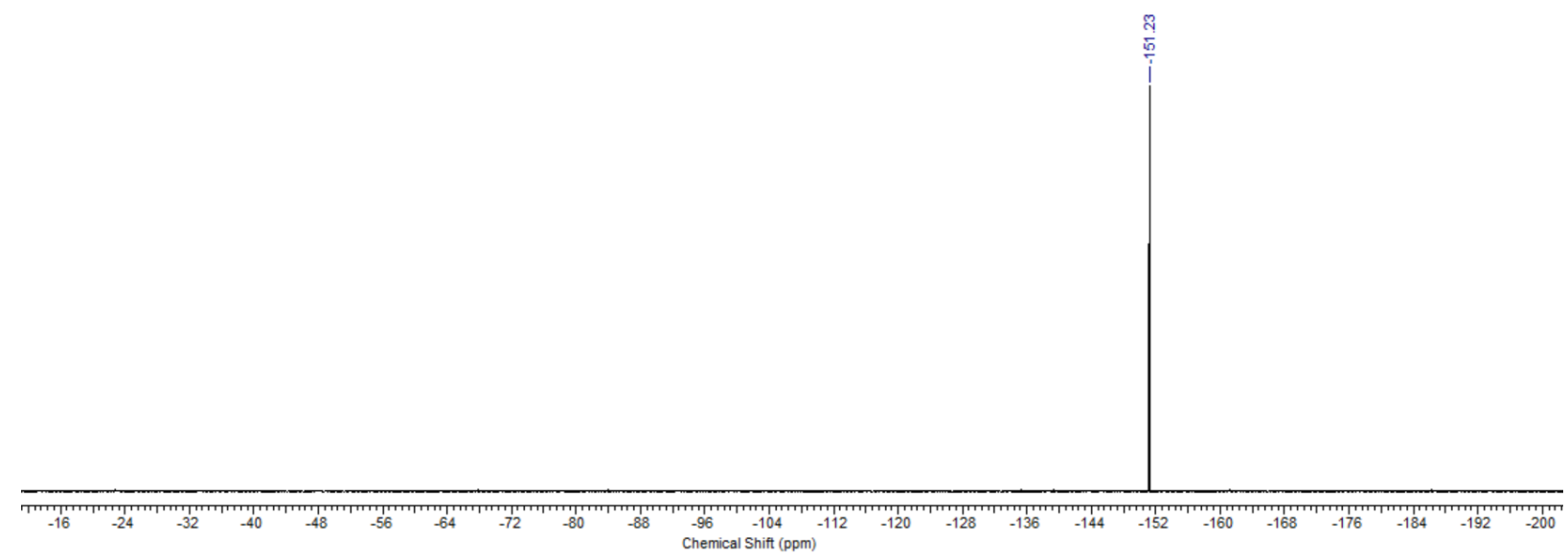

Figure S15. ${ }^{19} \mathrm{~F}$ NMR spectrum of $\mathbf{4 a},\left[\mathrm{PdI}\left(\mathrm{CH}_{2} \mathrm{CMe}_{2} \mathrm{C}_{6} \mathrm{H}_{4}\right)\left(\kappa^{3}-N, N^{\prime}, N^{\prime \prime}-\mathbf{L} 2\right)\right]\left[\mathrm{BF}_{4}\right]$, at $25^{\circ} \mathrm{C}(564$ $\left.\mathrm{MHz}, \mathrm{CDCl}_{3}\right)$.

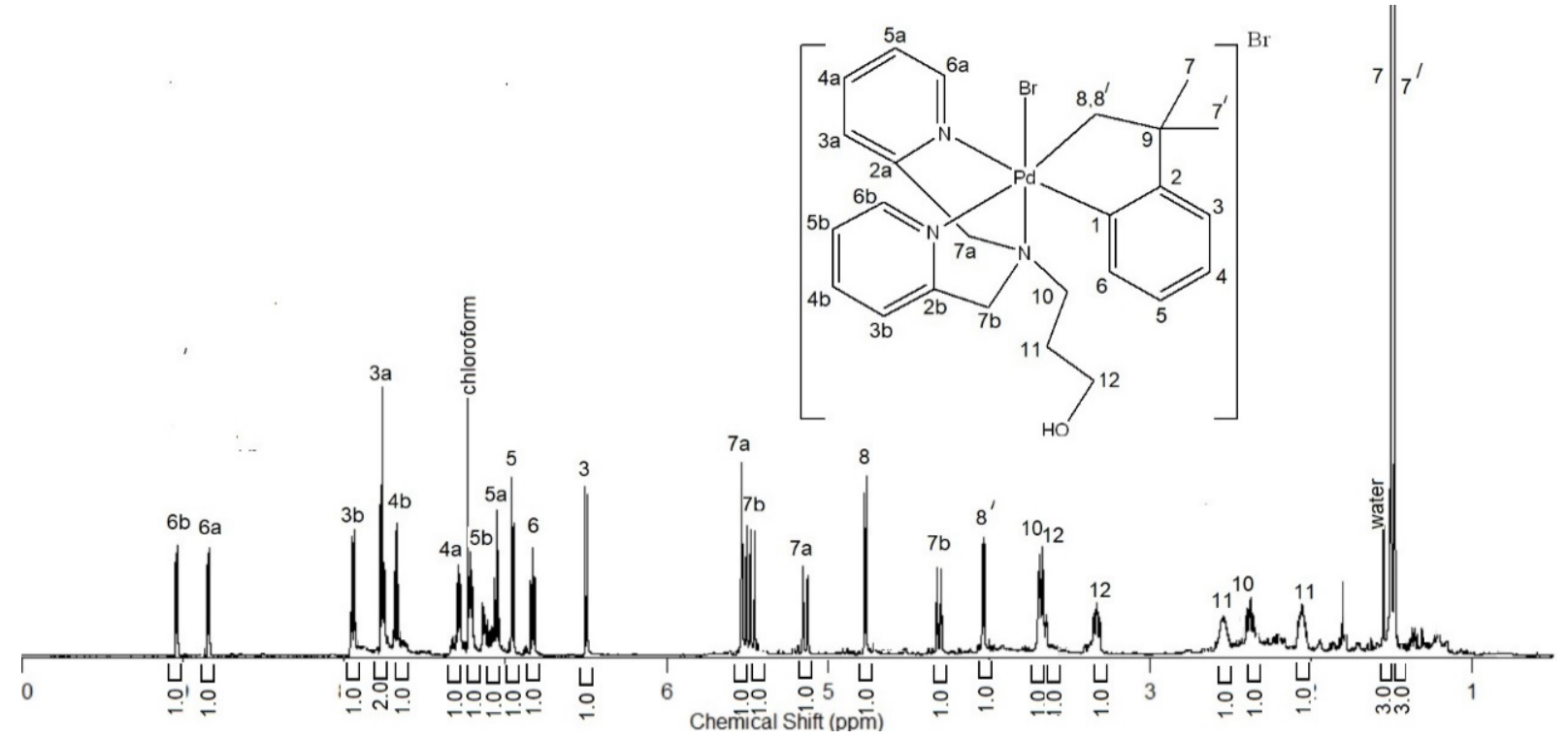

Figure S16. ${ }^{1} \mathrm{H}$ NMR spectrum of 5, $\left[\mathrm{PdBr}\left(\mathrm{CH}_{2} \mathrm{CMe}_{2} \mathrm{C}_{6} \mathrm{H}_{4}\right)\left(\kappa^{3}-N, N^{\prime}, N^{\prime \prime}-\mathbf{L 1}\right)\right][\mathrm{Br}]$, at $25^{\circ} \mathrm{C}(600$ $\left.\mathrm{MHz}, \mathrm{CDCl}_{3}\right)$. 


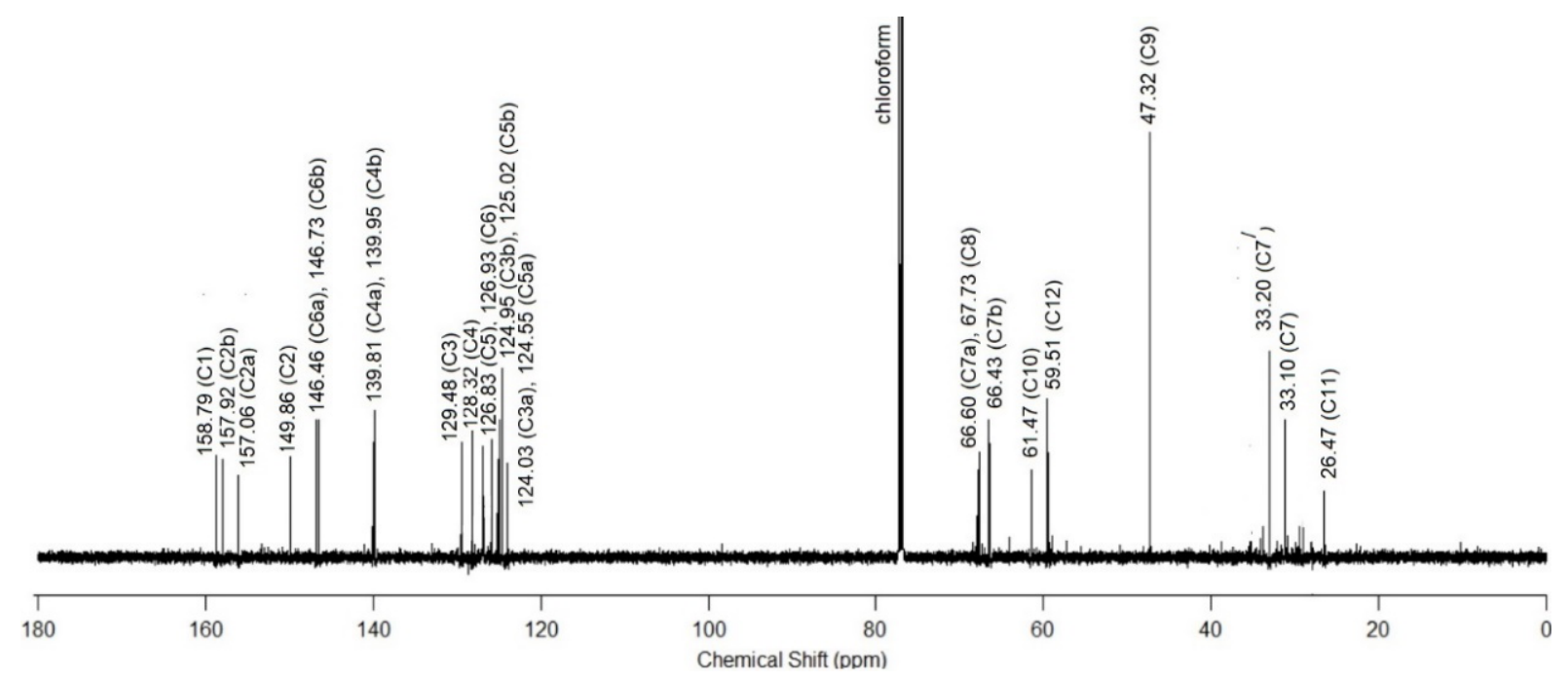

Figure S17. ${ }^{13} \mathrm{C}\left\{{ }^{1} \mathrm{H}\right\}$ NMR spectrum of 5, $\left[\mathrm{PdBr}\left(\mathrm{CH}_{2} \mathrm{CMe}_{2} \mathrm{C}_{6} \mathrm{H}_{4}\right)\left(\kappa^{3}-N, N^{\prime}, N^{\prime \prime}-\mathbf{L 1}\right)\right][\mathrm{Br}]$, at $25^{\circ} \mathrm{C}$ (151 $\left.\mathrm{MHz}, \mathrm{CDCl}_{3}\right)$.

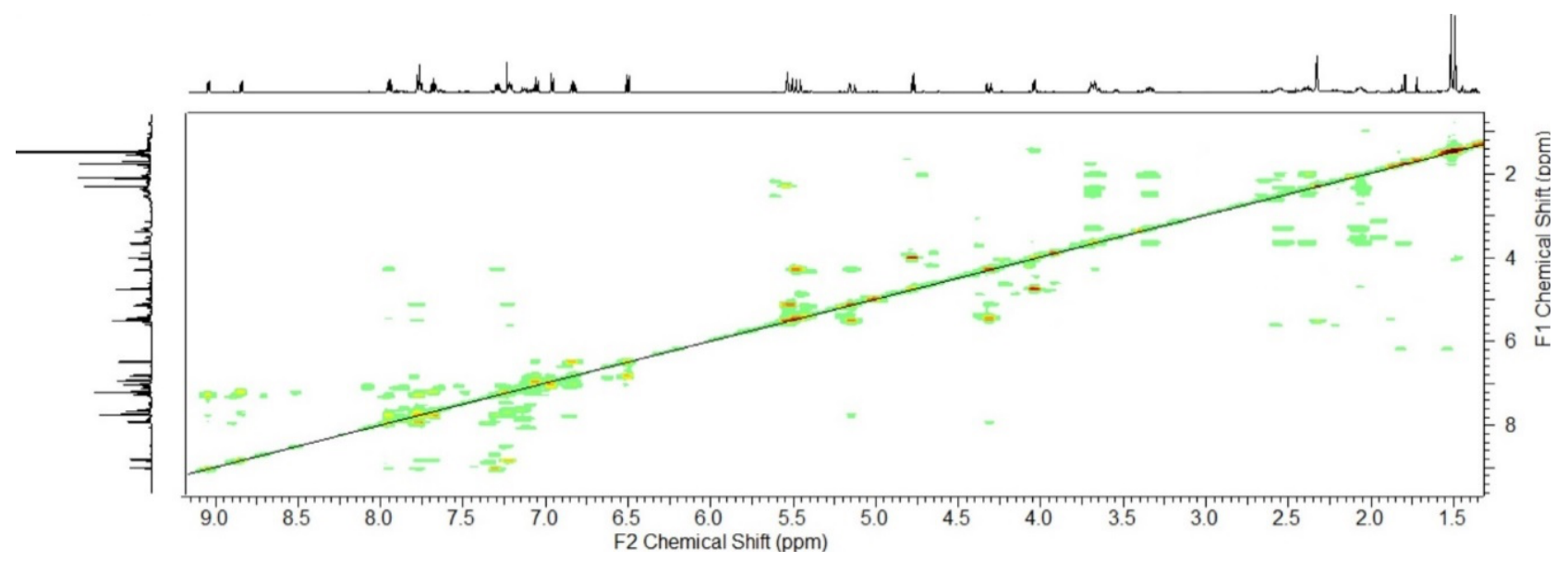

Figure S18. ${ }^{1} \mathrm{H}-{ }^{1} \mathrm{H}$ gCOSY NMR spectrum of 5, $\left[\mathrm{PdBr}\left(\mathrm{CH}_{2} \mathrm{CMe}_{2} \mathrm{C}_{6} \mathrm{H}_{4}\right)\left(\kappa^{3}-N, N^{\prime}, N^{\prime \prime}-\mathbf{L} \mathbf{1}\right)\right][\mathrm{Br}]$, at $25^{\circ} \mathrm{C}\left(600 \mathrm{MHz}, \mathrm{CDCl}_{3}\right)$. 


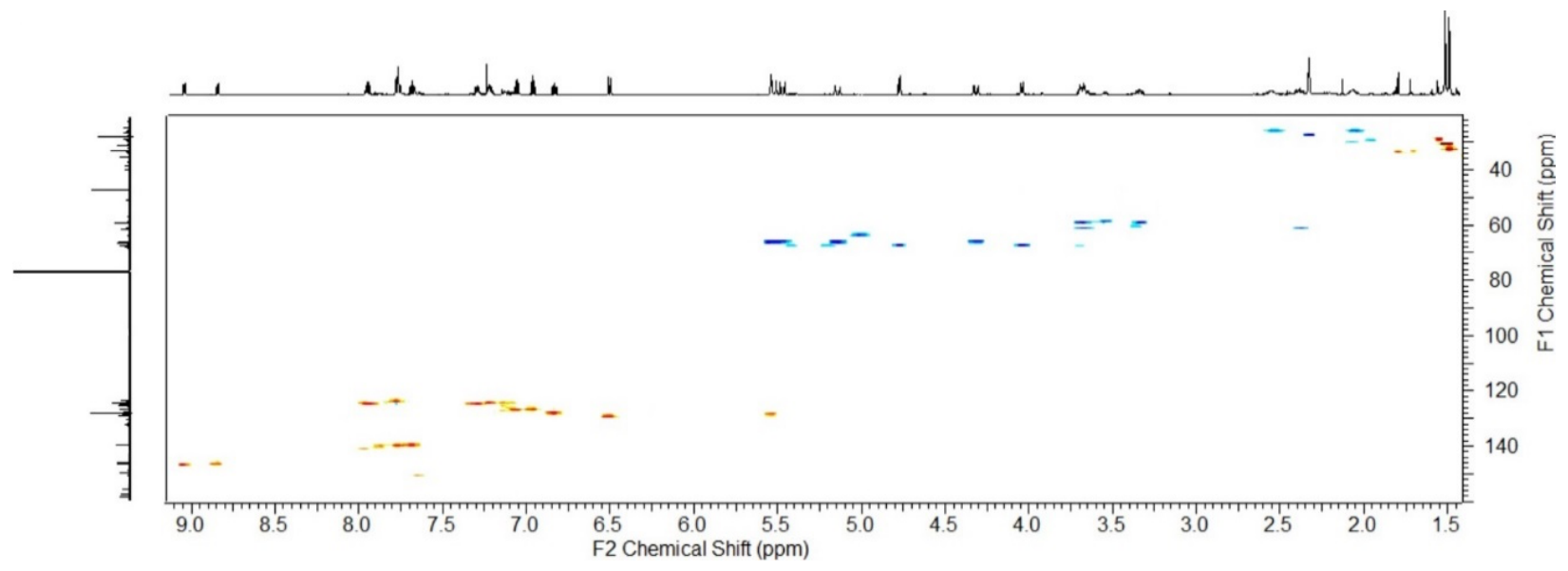

Figure S19. ${ }^{1} \mathrm{H}-{ }^{13} \mathrm{C}$ HSQC NMR spectrum of 5, $\left[\mathrm{PdBr}\left(\mathrm{CH}_{2} \mathrm{CMe}_{2} \mathrm{C}_{6} \mathrm{H}_{4}\right)\left(\kappa^{3}-N, N^{\prime}, N^{\prime \prime}-\mathbf{L 1}\right)\right][\mathrm{Br}]$, at $25^{\circ} \mathrm{C}\left(600 \mathrm{MHz}, \mathrm{CDCl}_{3}\right)$.

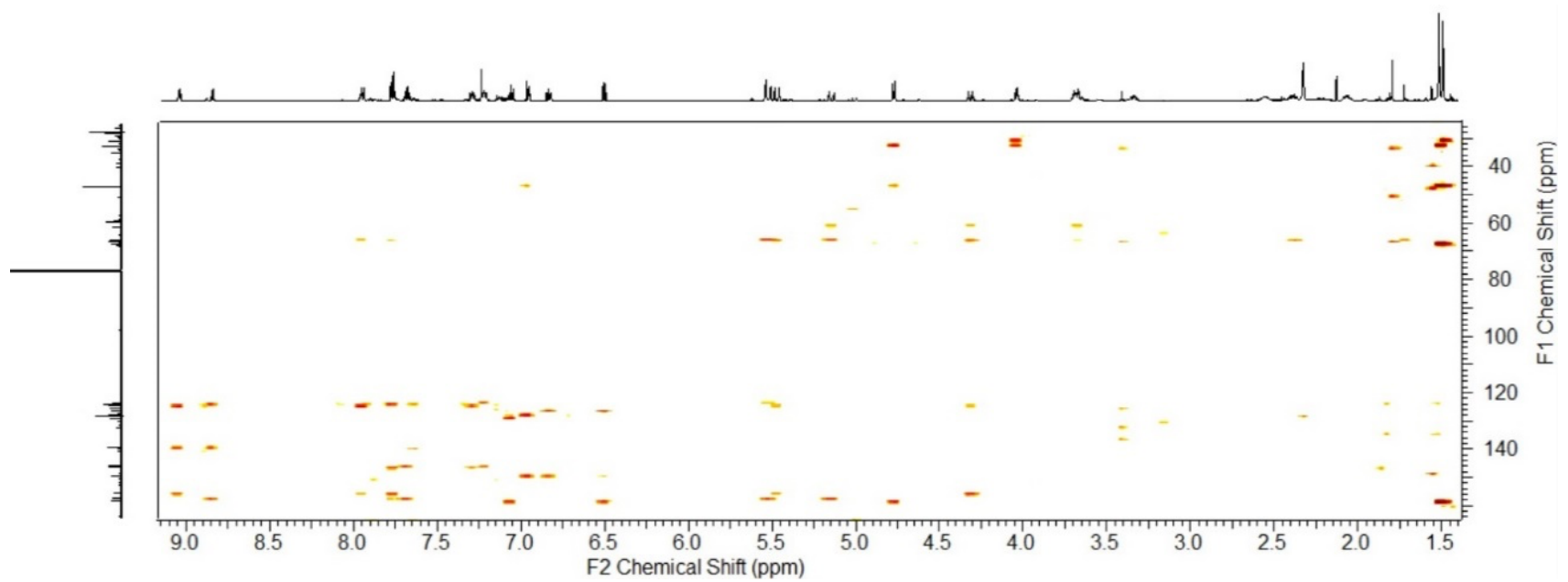

Figure S20. ${ }^{1} \mathrm{H}-{ }^{13} \mathrm{C}$ HMBC NMR spectrum of 5, $\left[\mathrm{PdBr}\left(\mathrm{CH}_{2} \mathrm{CMe}_{2} \mathrm{C}_{6} \mathrm{H}_{4}\right)\left(\kappa^{3}-N, N^{\prime}, N^{\prime \prime}-\mathbf{L} \mathbf{1}\right)\right][\mathrm{Br}]$, at $25^{\circ} \mathrm{C}\left(600 \mathrm{MHz}, \mathrm{CDCl}_{3}\right)$. 


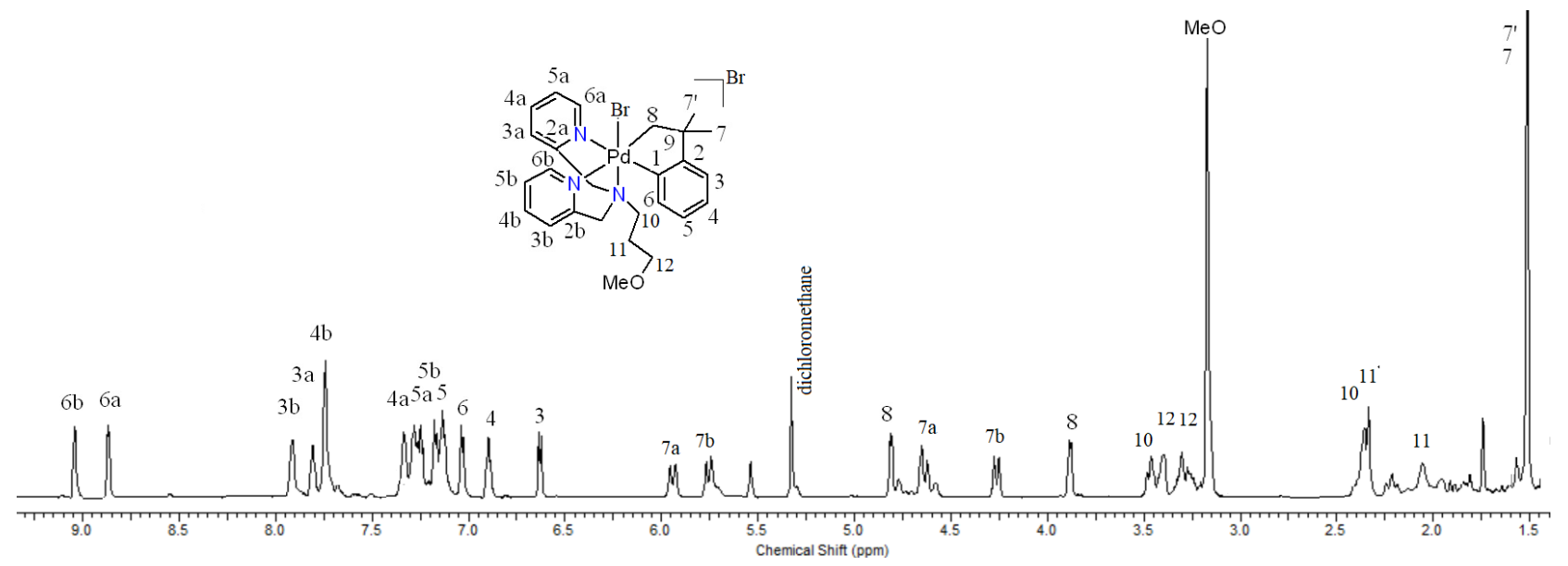

Figure S21. ${ }^{1} \mathrm{H}$ NMR spectrum of 6, $\left[\mathrm{PdBr}\left(\mathrm{CH}_{2} \mathrm{CMe}_{2} \mathrm{C}_{6} \mathrm{H}_{4}\right)\left(\kappa^{3}-N, N^{\prime}, N^{\prime \prime}-\mathbf{L} 2\right)\right][\mathrm{Br}]$, at $-8{ }^{\circ} \mathrm{C}(600$ $\mathrm{MHz}, \mathrm{CD}_{2} \mathrm{Cl}_{2}$ ).

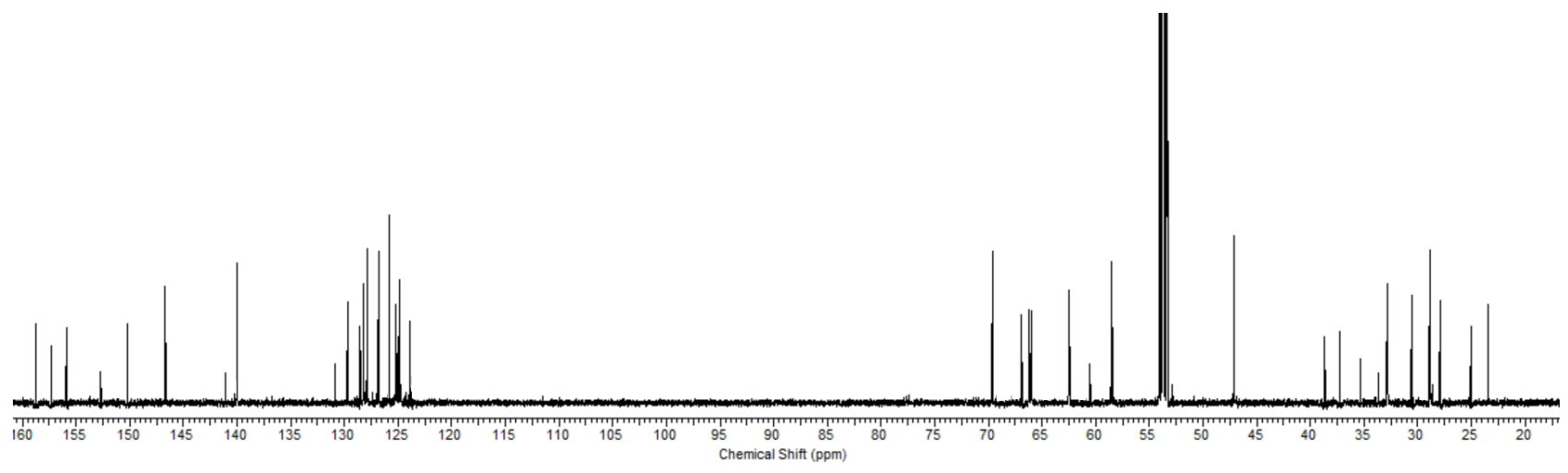

Figure S22. ${ }^{13} \mathrm{C}\left\{{ }^{1} \mathrm{H}\right\}$ NMR spectrum of 6, $\left[\mathrm{PdBr}\left(\mathrm{CH}_{2} \mathrm{CMe}_{2} \mathrm{C}_{6} \mathrm{H}_{4}\right)\left(\kappa^{3}-N, N^{\prime}, N^{\prime \prime}-\mathbf{L} \mathbf{2}\right)\right][\mathrm{Br}]$, at $-8{ }^{\circ} \mathrm{C}$ $\left(151 \mathrm{MHz}, \mathrm{CD}_{2} \mathrm{Cl}_{2}\right.$ ). 


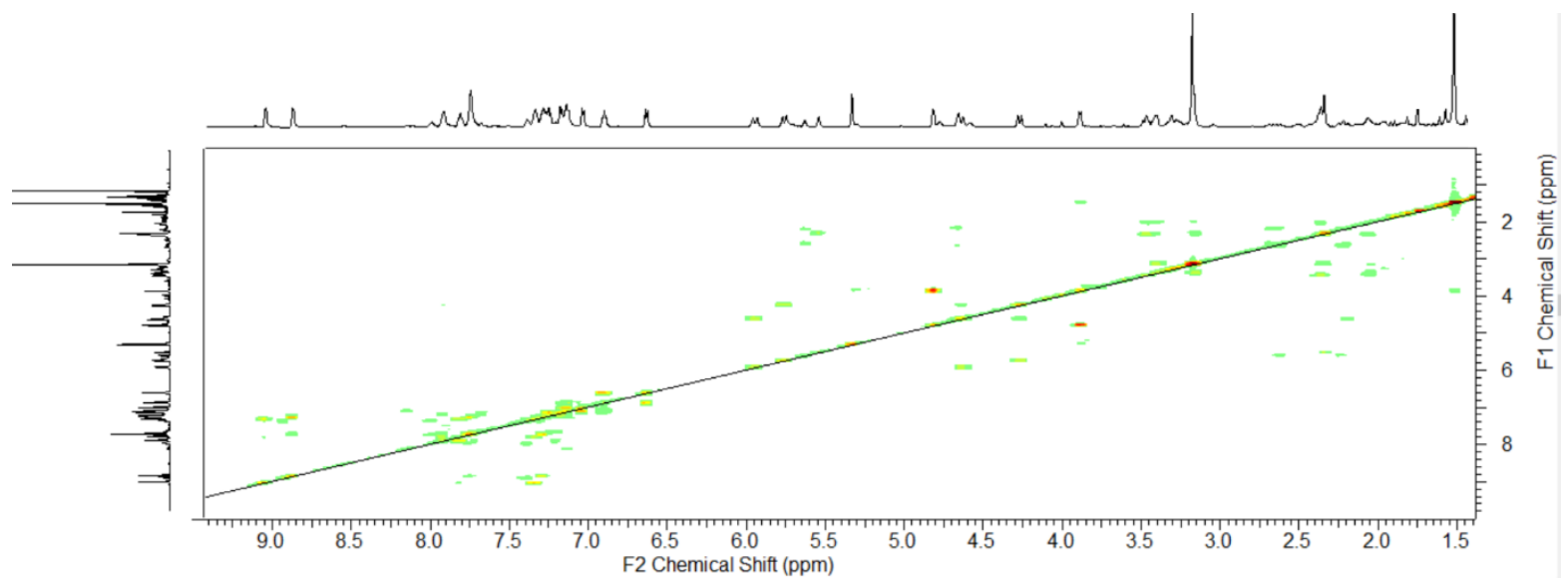

Figure S23. ${ }^{1} \mathrm{H}-{ }^{1} \mathrm{H}$ gCOSY NMR spectrum of 6, $\left[\mathrm{PdBr}\left(\mathrm{CH}_{2} \mathrm{CMe}_{2} \mathrm{C}_{6} \mathrm{H}_{4}\right)\left(\kappa^{3}-N, N^{\prime}, N^{\prime \prime}-\mathbf{L} 2\right)\right][\mathrm{Br}]$, acquired at $-8{ }^{\circ} \mathrm{C}\left(600 \mathrm{MHz}, \mathrm{CD}_{2} \mathrm{Cl}_{2}\right)$.

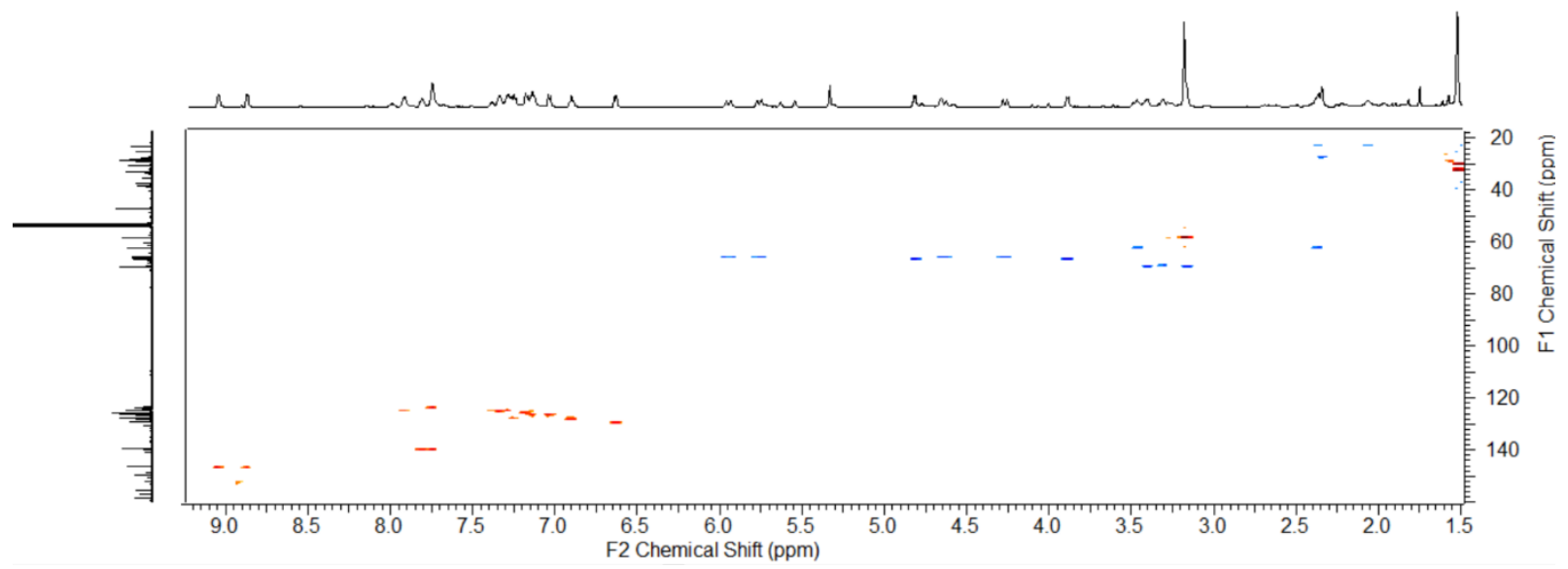

Figure S24. ${ }^{1} \mathrm{H}-{ }^{13} \mathrm{C}$ HSQC NMR spectrum of 6, $\left[\mathrm{PdBr}\left(\mathrm{CH}_{2} \mathrm{CMe}_{2} \mathrm{C}_{6} \mathrm{H}_{4}\right)\left(\kappa^{3}-N, N^{\prime}, N^{\prime \prime}-\mathbf{L 2}\right)\right][\mathrm{Br}]$, acquired at $-8{ }^{\circ} \mathrm{C}\left(600 \mathrm{MHz}, \mathrm{CD}_{2} \mathrm{Cl}_{2}\right)$. 


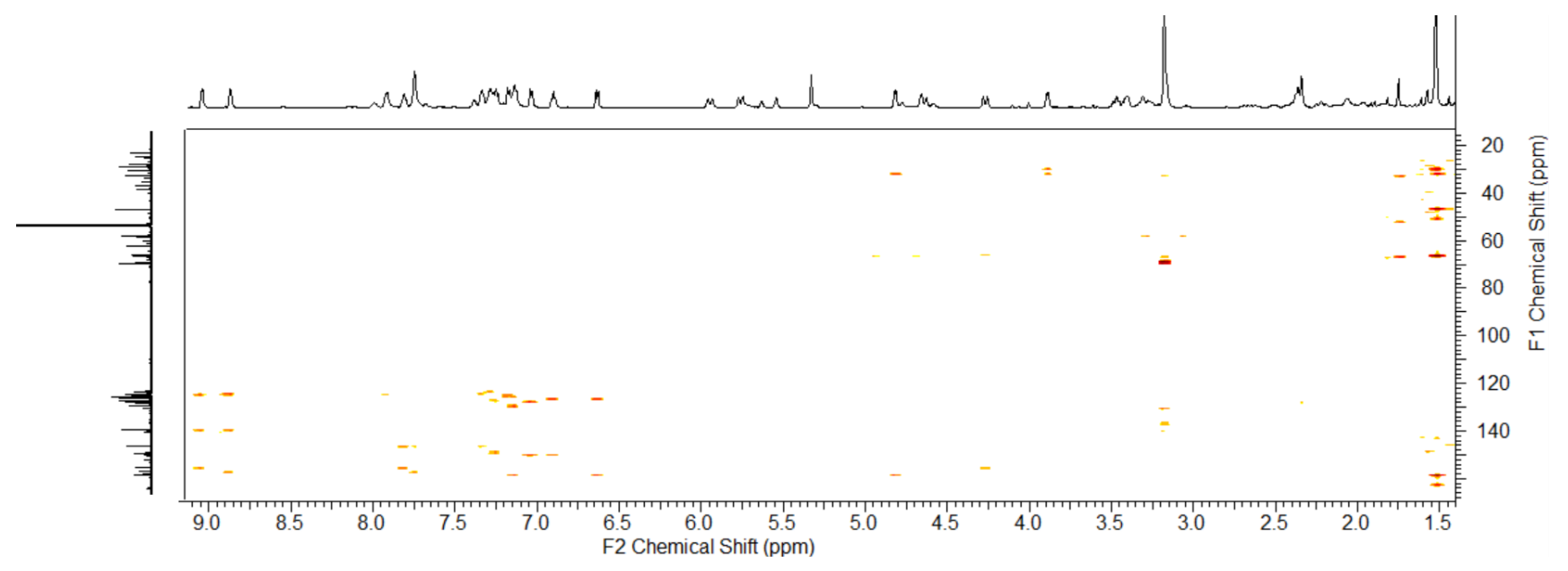

Figure S25. ${ }^{1} \mathrm{H}-{ }^{13} \mathrm{C}$ HMBC NMR spectrum of 6, $\left[\mathrm{PdBr}\left(\mathrm{CH}_{2} \mathrm{CMe}_{2} \mathrm{C}_{6} \mathrm{H}_{4}\right)\left(\kappa^{3}-N, N^{\prime}, N^{\prime \prime}-\mathbf{L} 2\right)\right][\mathrm{Br}]$, acquired at $-8{ }^{\circ} \mathrm{C}\left(600 \mathrm{MHz}, \mathrm{CD}_{2} \mathrm{Cl}_{2}\right)$.

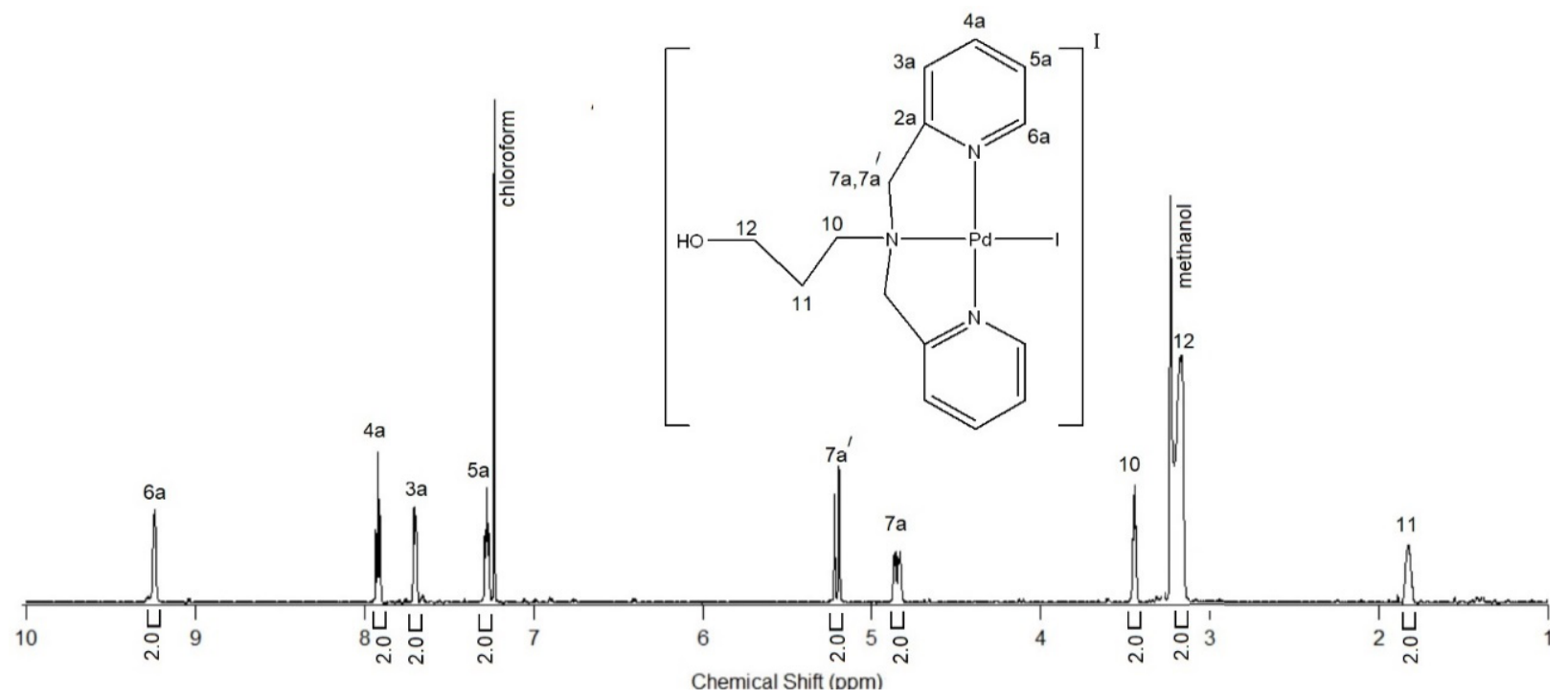

Figure S26. ${ }^{1} \mathrm{H}$ NMR spectrum of 7, $\left[\mathrm{PdI}\left(\kappa^{3}-N, N^{\prime}, N^{\prime \prime}-\mathbf{L 1}\right)\right][\mathrm{I}]$, at $25{ }^{\circ} \mathrm{C}(600 \mathrm{MHz}, 1: 0.3$ $\left.\mathrm{CDCl}_{3}: \mathrm{CD}_{3} \mathrm{OD}\right)$. 


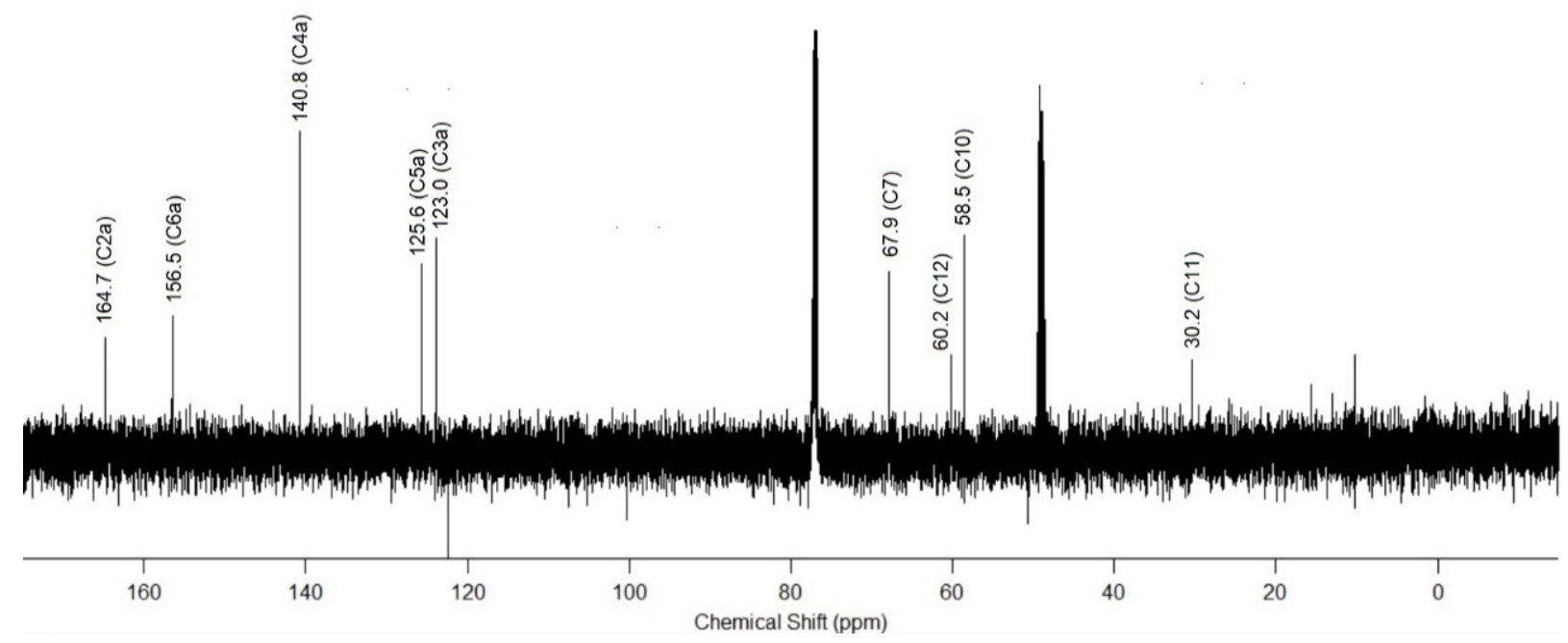

Figure S27. ${ }^{13} \mathrm{C}\left\{{ }^{1} \mathrm{H}\right\}$ NMR spectrum of 7, $\left[\mathrm{PdI}\left(\kappa^{3}-N, N^{\prime}, N^{\prime \prime}-\mathbf{L 1}\right)\right][\mathrm{I}]$, at $25{ }^{\circ} \mathrm{C}(151 \mathrm{MHz}, 1: 0.3$ $\left.\mathrm{CDCl}_{3}: \mathrm{CD}_{3} \mathrm{OD}\right)$.

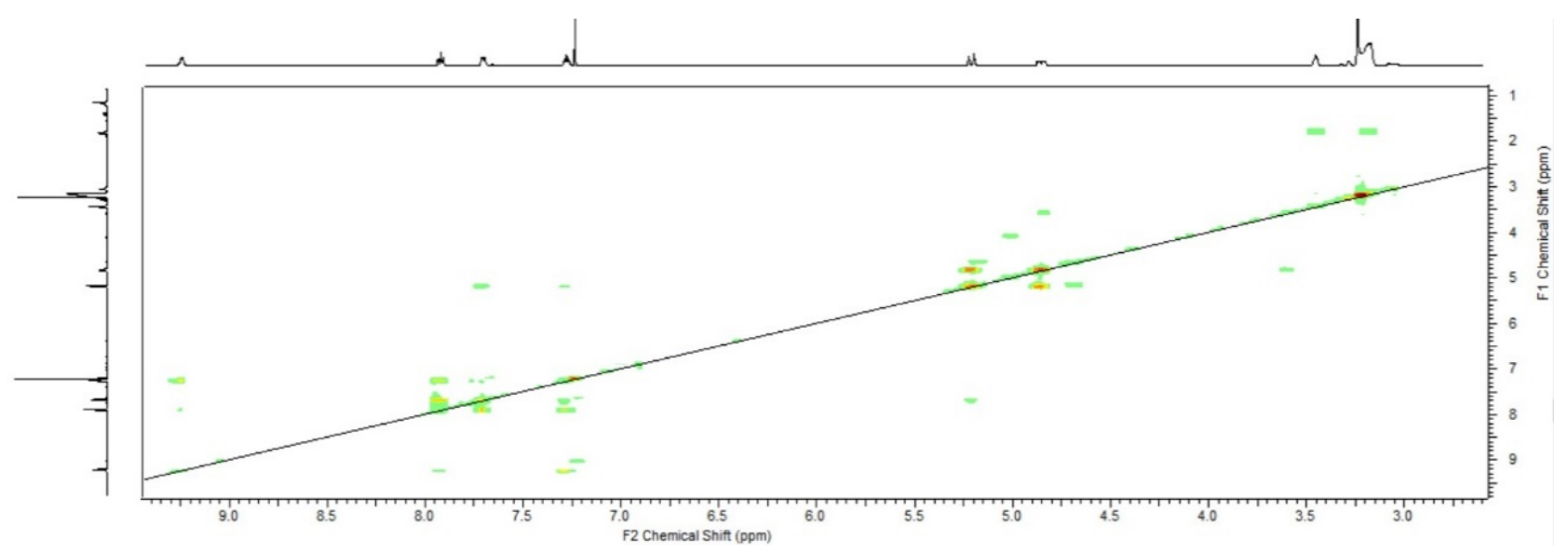

Figure S28. ${ }^{1} \mathrm{H}-{ }^{1} \mathrm{H}$ gCOSY NMR spectrum of 7, $\left[\mathrm{PdI}\left(\kappa^{3}-N, N^{\prime}, N^{\prime \prime}-\mathbf{L} \mathbf{1}\right)\right][\mathrm{I}]$, at $25^{\circ} \mathrm{C}(600 \mathrm{MHz}$, $\left.1: 0.3 \mathrm{CDCl}_{3}: \mathrm{CD}_{3} \mathrm{OD}\right)$. 


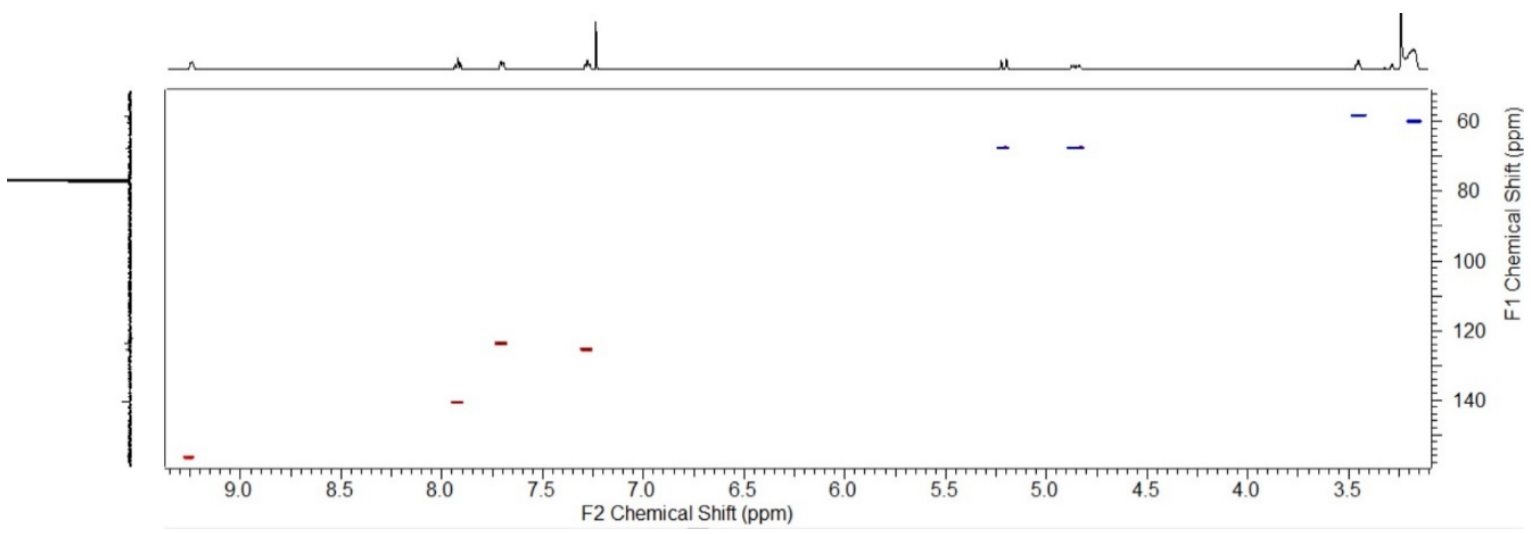

Figure S29. ${ }^{1} \mathrm{H}-{ }^{13} \mathrm{C}$ HSQC NMR spectrum of 7, $\left[\mathrm{PdI}\left(\kappa^{3}-N, N^{\prime}, N^{\prime \prime}-\mathbf{L 1}\right)\right][\mathrm{I}]$, at $25{ }^{\circ} \mathrm{C}(600 \mathrm{MHz}, 1: 0.3$ $\left.\mathrm{CDCl}_{3}: \mathrm{CD}_{3} \mathrm{OD}\right)$.

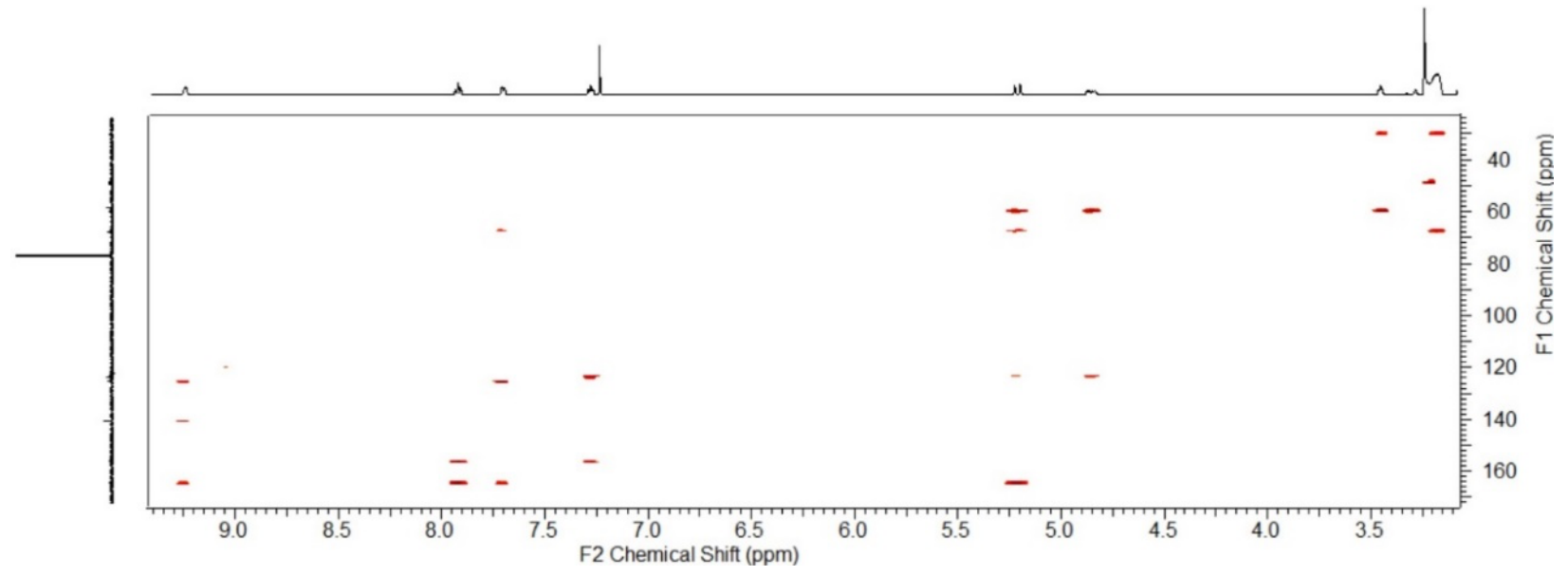

Figure S30. ${ }^{1} \mathrm{H}-{ }^{13} \mathrm{C}$ HSQC NMR spectrum of 7, $\left[\mathrm{PdI}\left(\kappa^{3}-N, N^{\prime}, N^{\prime \prime}-\mathbf{L} \mathbf{1}\right)\right][\mathrm{I}]$, at $25{ }^{\circ} \mathrm{C}(600 \mathrm{MHz}, 1: 0.3$ $\left.\mathrm{CDCl}_{3}: \mathrm{CD}_{3} \mathrm{OD}\right)$. 


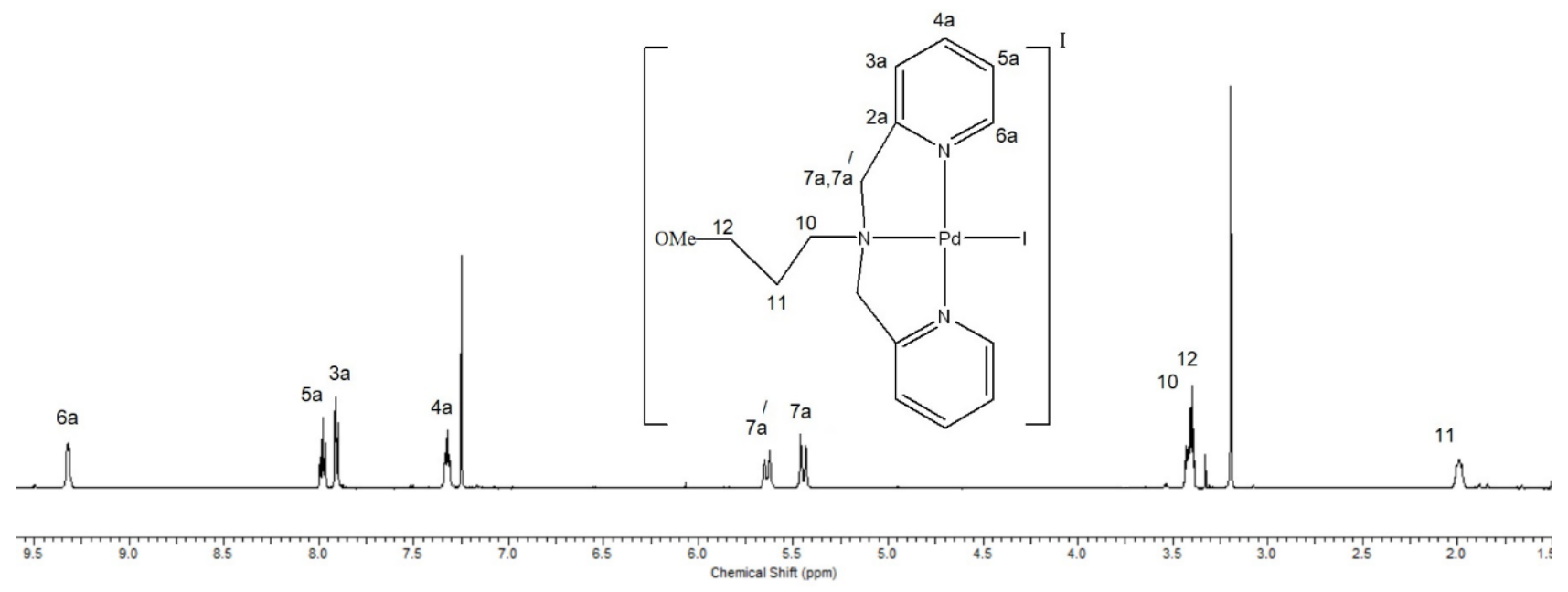

Figure S31. ${ }^{1} \mathrm{H}$ NMR spectrum of 8, $\left[\mathrm{PdI}\left(\kappa^{3}-N, N^{\prime}, N^{\prime \prime}-\mathbf{L 2}\right)\right][\mathrm{I}]$, at $25{ }^{\circ} \mathrm{C}\left(600 \mathrm{MHz}, \mathrm{CDCl}_{3}\right)$.

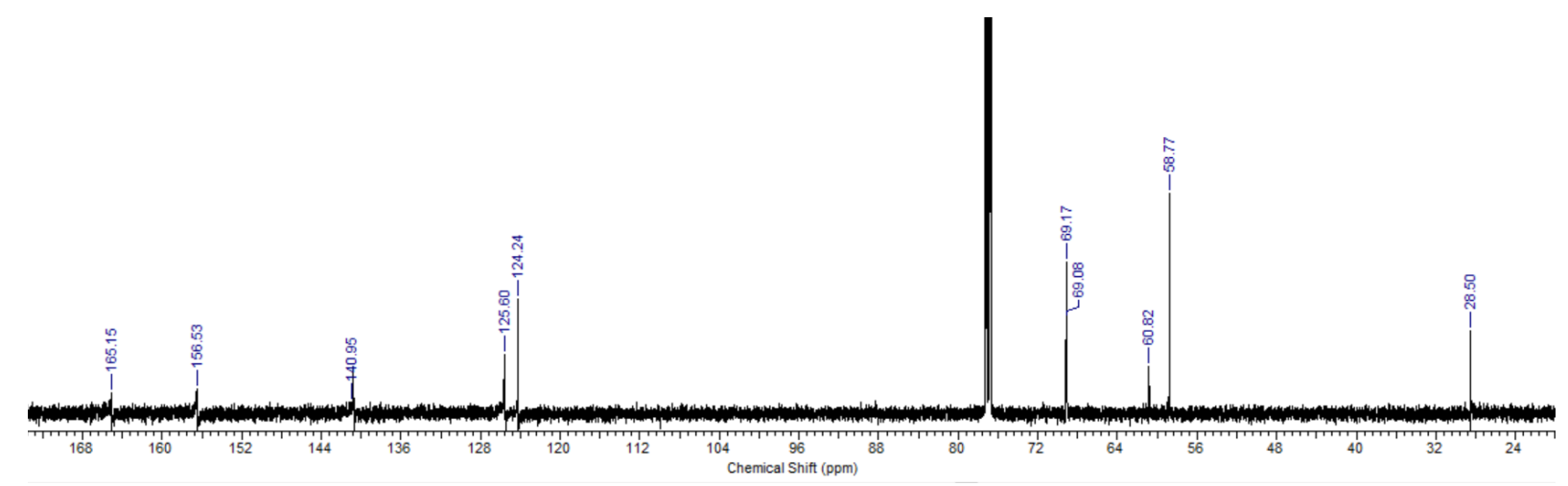

Figure S32. ${ }^{13} \mathrm{C}\left\{{ }^{1} \mathrm{H}\right\} \mathrm{NMR}$ spectrum of 8, $\left[\mathrm{PdI}\left(\kappa^{3}-N, N^{\prime}, N^{\prime \prime}-\mathbf{L 2}\right)\right][\mathrm{I}]$, at $25{ }^{\circ} \mathrm{C}\left(151 \mathrm{MHz}, \mathrm{CDCl}_{3}\right)$. 


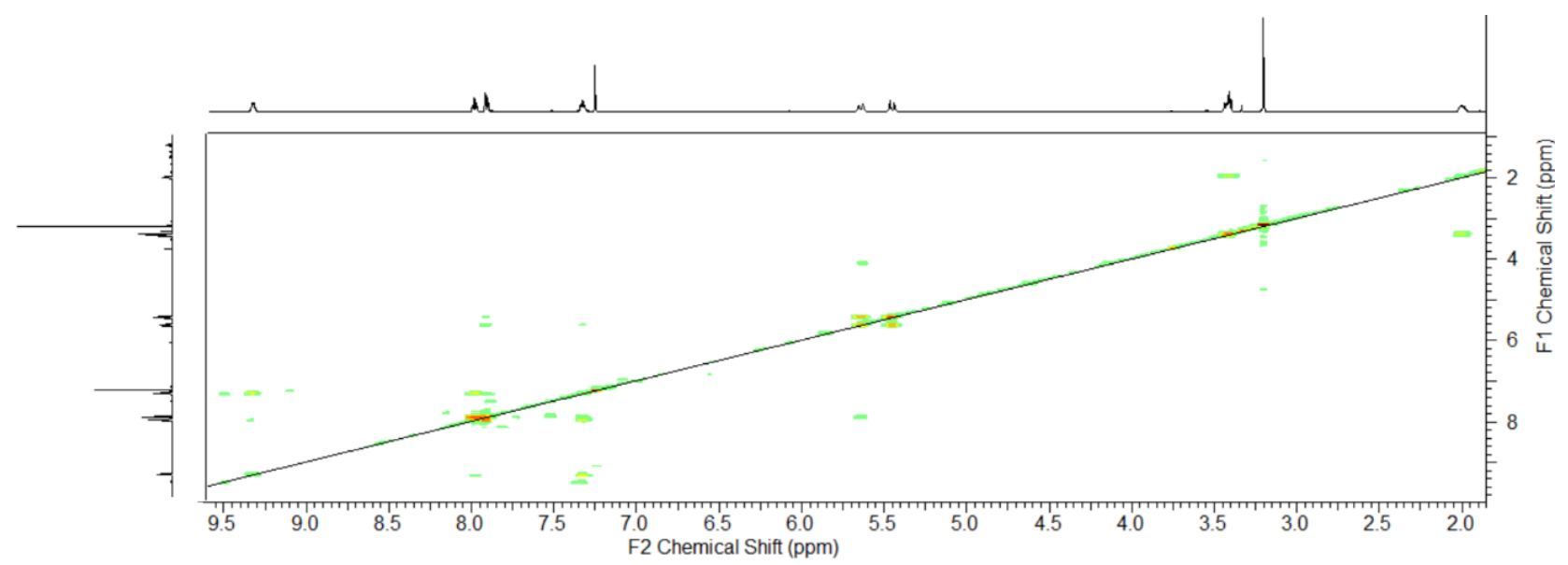

Figure S33. ${ }^{1} \mathrm{H}-{ }^{1} \mathrm{H}$ gCOSY NMR spectrum of 8, $\left[\mathrm{PdI}\left(\kappa^{3}-N, N^{\prime}, N^{\prime \prime}-\mathbf{L} 2\right)\right][\mathrm{I}]$, at $25{ }^{\circ} \mathrm{C}(600 \mathrm{MHz}$, $\left.\mathrm{CDCl}_{3}\right)$.

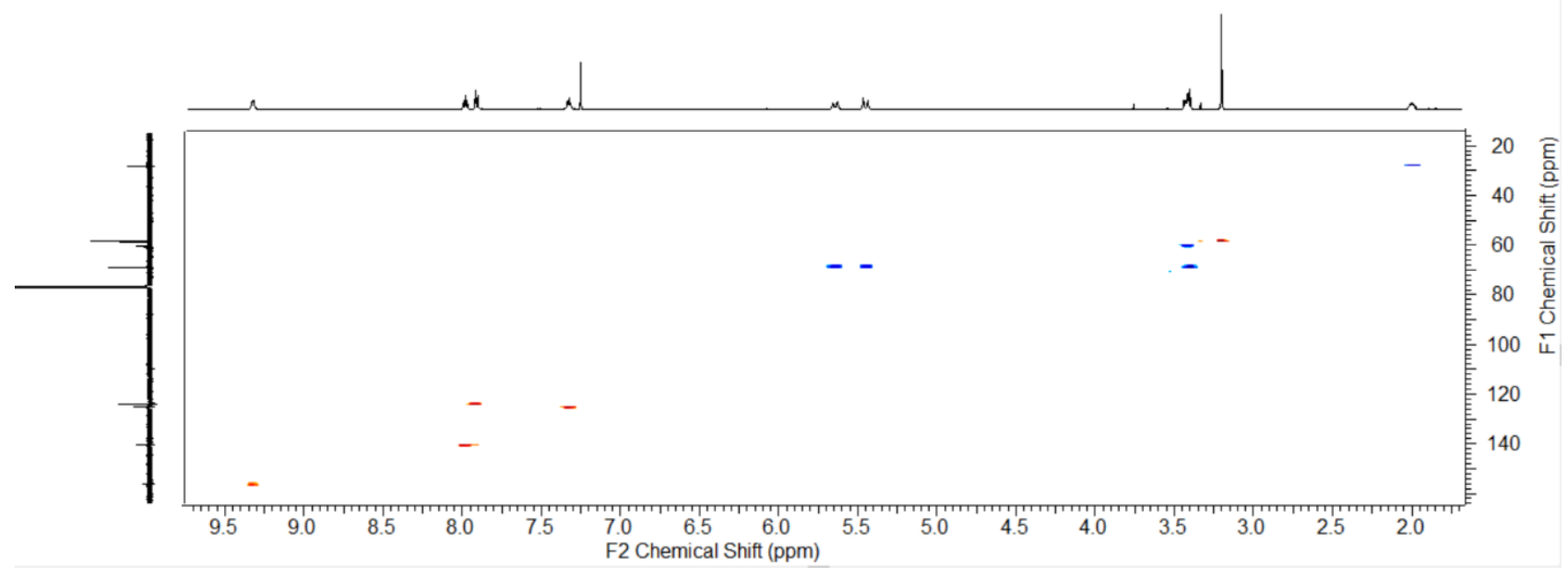

Figure S34. ${ }^{1} \mathrm{H}-{ }^{13} \mathrm{C}$ HSQC NMR spectrum of 8, $\left[\mathrm{PdI}\left(\kappa^{3}-N, N^{\prime}, N^{\prime \prime}-\mathbf{L} 2\right)\right][\mathrm{I}]$, at $25^{\circ} \mathrm{C}(600 \mathrm{MHz}$, $\left.\mathrm{CDCl}_{3}\right)$. 


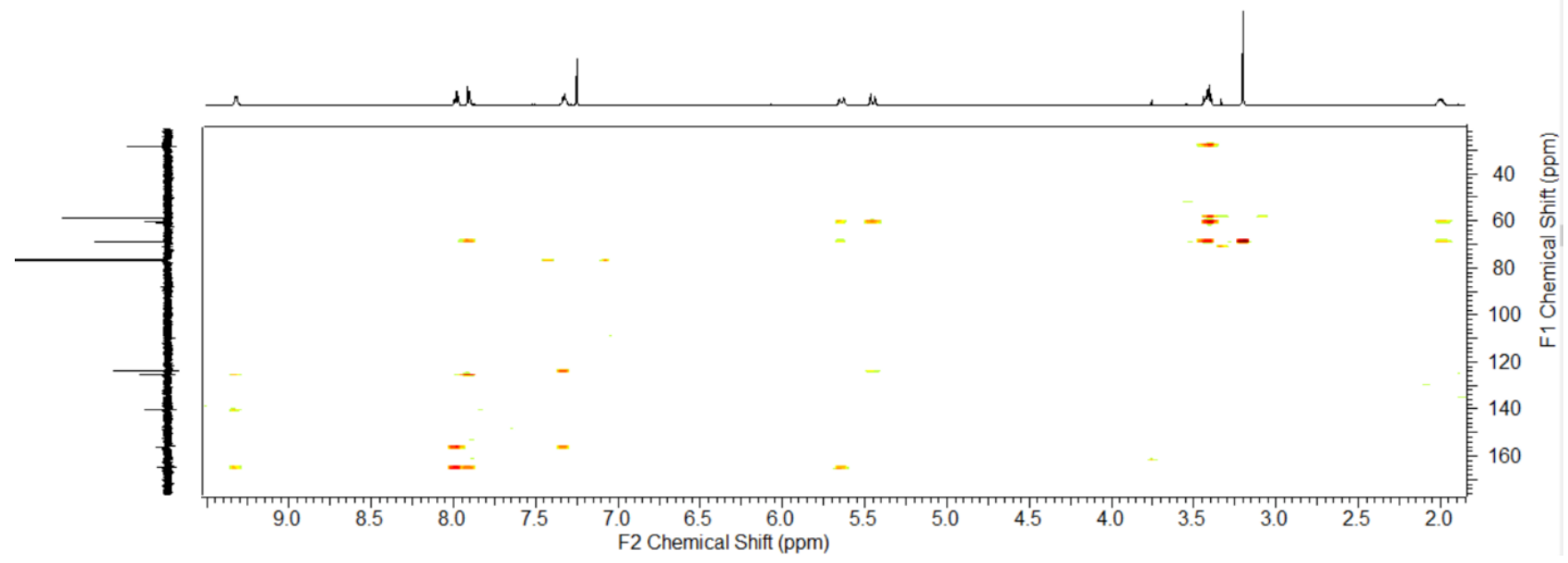

Figure S35. ${ }^{1} \mathrm{H}_{-}{ }^{13} \mathrm{C}$ HMBC NMR spectrum of 8, $\left[\mathrm{PdI}\left(\kappa^{3}-N, N^{\prime}, N^{\prime \prime}-\mathbf{L} 2\right)\right][\mathrm{I}]$, at $25{ }^{\circ} \mathrm{C}(600 \mathrm{MHz}$, $\left.\mathrm{CDCl}_{3}\right)$.

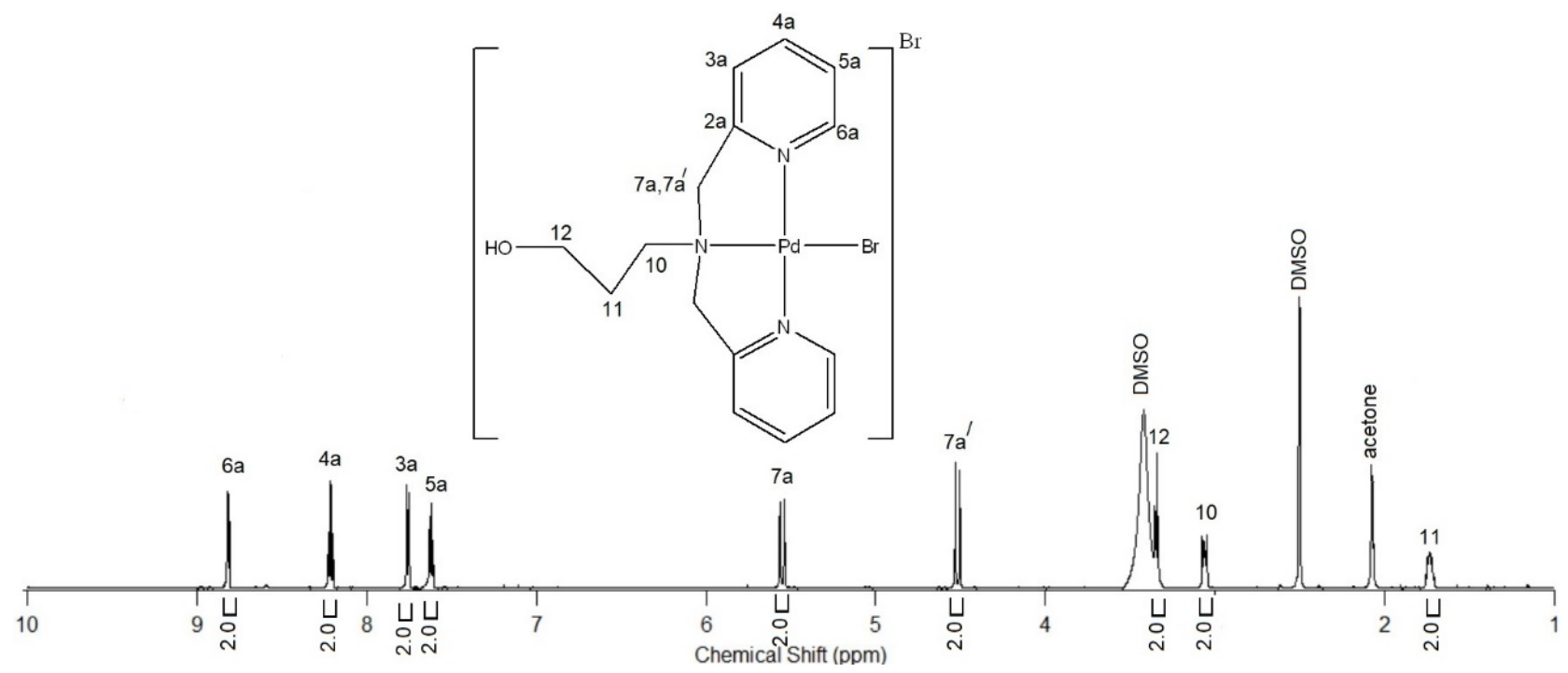

Figure S36. ${ }^{1} \mathrm{H}$ NMR spectrum of 9, $\left[\mathrm{PdBr}\left(\kappa^{3}-N, N^{\prime}, N^{\prime \prime}-\mathbf{L 1}\right)\right][\mathrm{Br}]$, at $25^{\circ} \mathrm{C}\left(600 \mathrm{MHz},\left(\mathrm{CD}_{3}\right)_{2} \mathrm{SO}\right)$. 


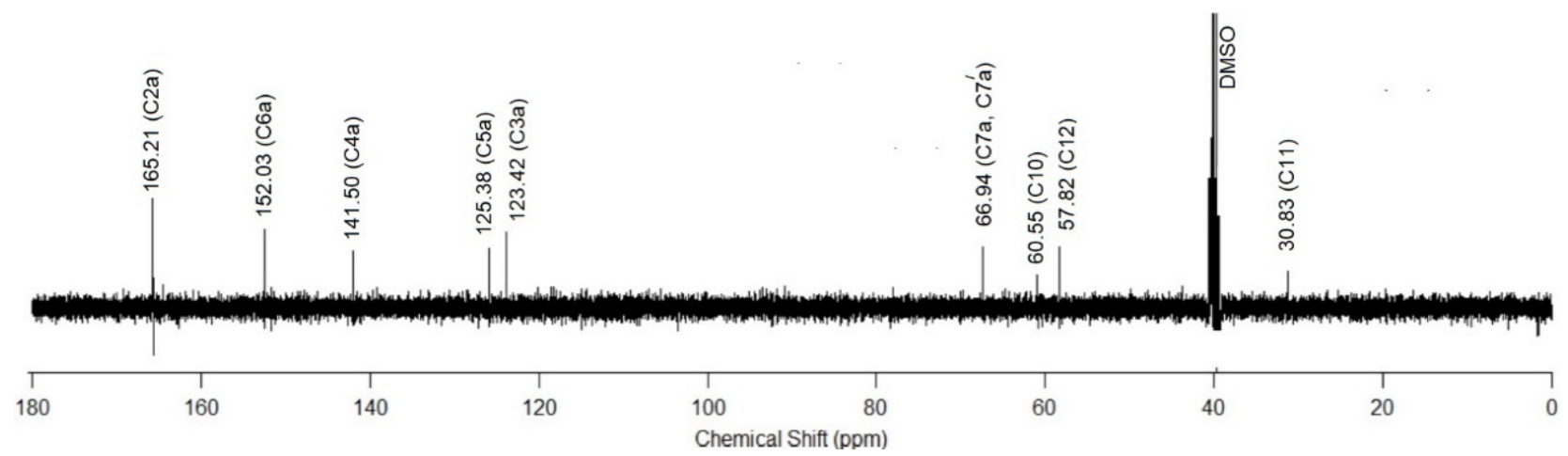

Figure S37. ${ }^{13} \mathrm{C}\left\{{ }^{1} \mathrm{H}\right\}$ NMR spectrum of 9, $\left[\mathrm{PdBr}\left(\kappa^{3}-N, N^{\prime}, N^{\prime \prime}-\mathbf{L 1}\right)\right][\mathrm{Br}]$, at $25{ }^{\circ} \mathrm{C}(151 \mathrm{MHz}$, $\left.\left(\mathrm{CD}_{3}\right)_{2} \mathrm{SO}\right)$.

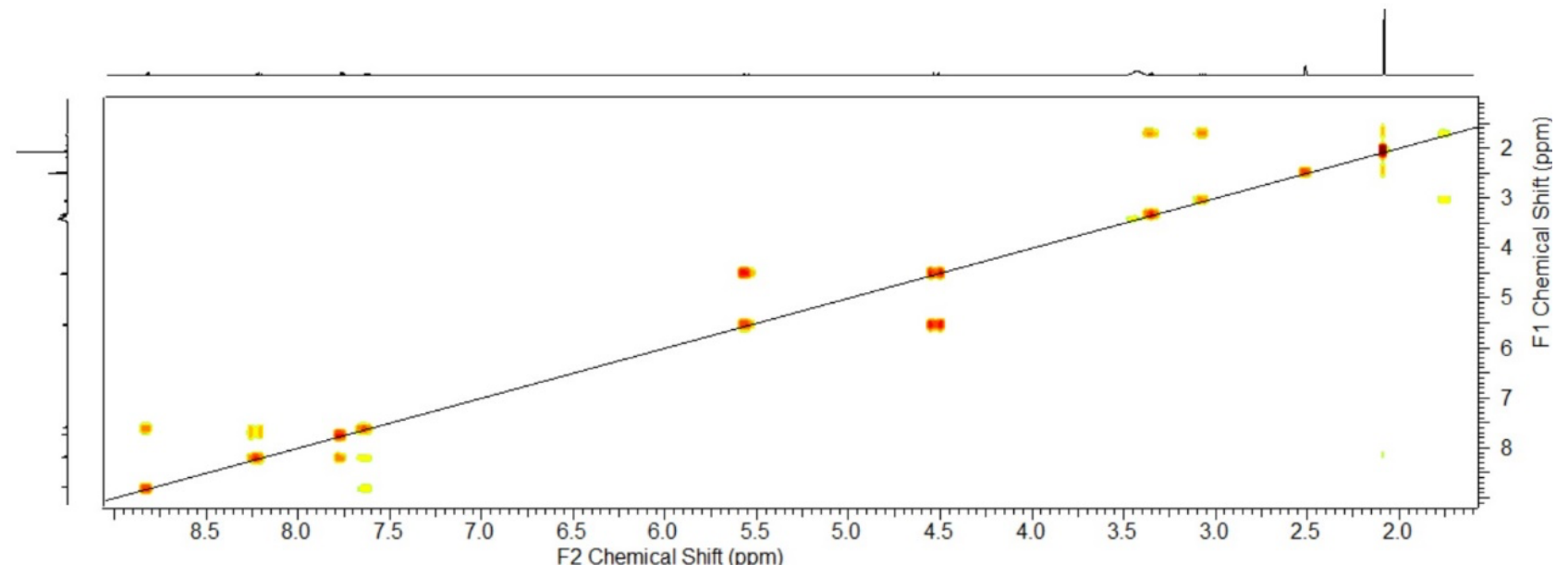

Figure S38. ${ }^{1} \mathrm{H}-{ }^{1} \mathrm{H}$ gCOSY NMR spectrum of 9, $\left[\mathrm{PdBr}\left(\kappa^{3}-N, N^{\prime}, N^{\prime \prime}-\mathbf{L} \mathbf{1}\right)\right][\mathrm{Br}]$, at $25^{\circ} \mathrm{C}(600 \mathrm{MHz}$, $\left.\left(\mathrm{CD}_{3}\right)_{2} \mathrm{SO}\right)$. 


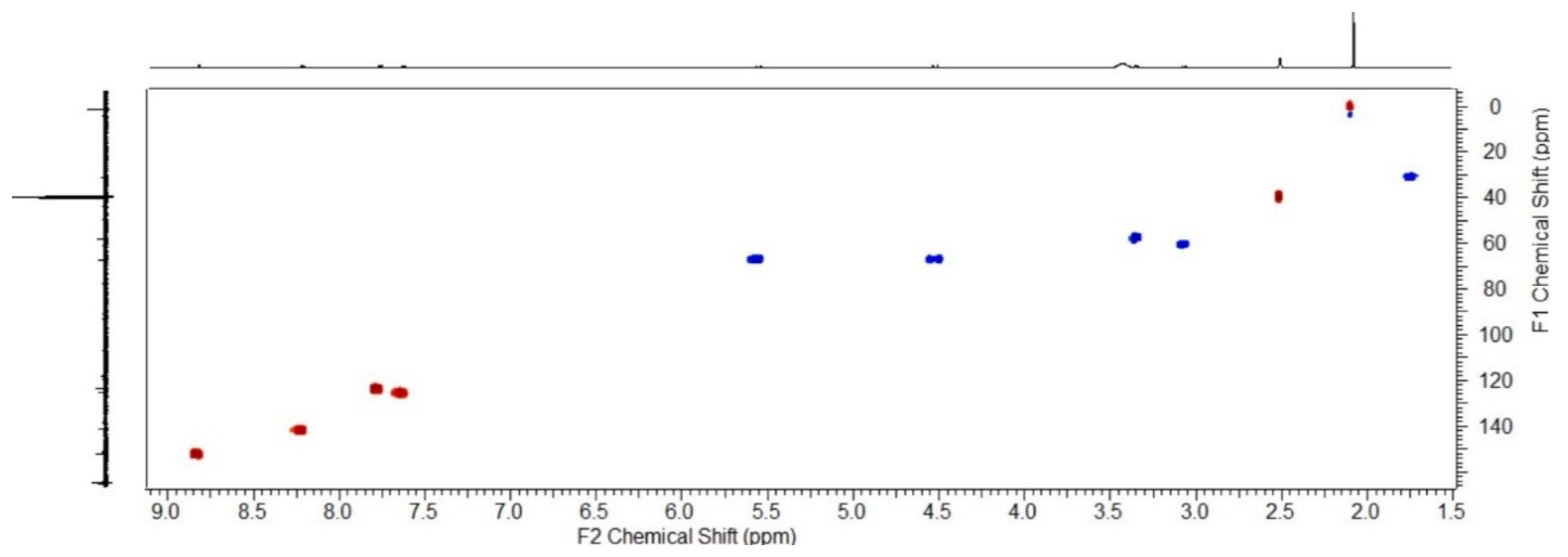

Figure S39. ${ }^{1} \mathrm{H}-{ }^{13} \mathrm{C}$ HSQC NMR spectrum of 9, $\left[\mathrm{PdBr}\left(\kappa^{3}-N, N^{\prime}, N^{\prime \prime}-\mathbf{L} \mathbf{1}\right)\right][\mathrm{Br}]$, at $25{ }^{\circ} \mathrm{C}(600 \mathrm{MHz}$, $\left.\left(\mathrm{CD}_{3}\right)_{2} \mathrm{SO}\right)$.

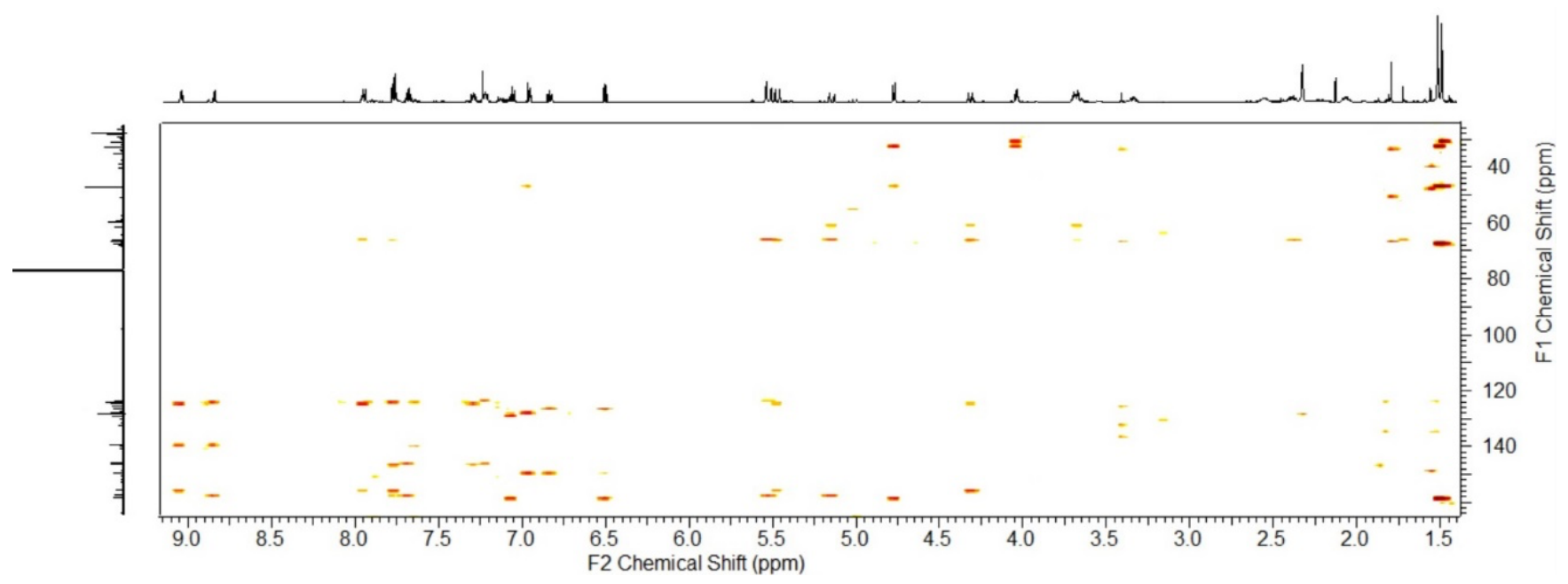

Figure S40. ${ }^{1} \mathrm{H}-{ }^{13} \mathrm{C} \mathrm{HMBC}$ NMR spectrum of 9, $\left[\mathrm{PdBr}\left(\kappa^{3}-N, N^{\prime}, N^{\prime \prime}-\mathbf{L 1}\right)\right][\mathrm{Br}]$, at $25^{\circ} \mathrm{C}(600 \mathrm{MHz}$, $\left.\left(\mathrm{CD}_{3}\right)_{2} \mathrm{SO}\right)$. 


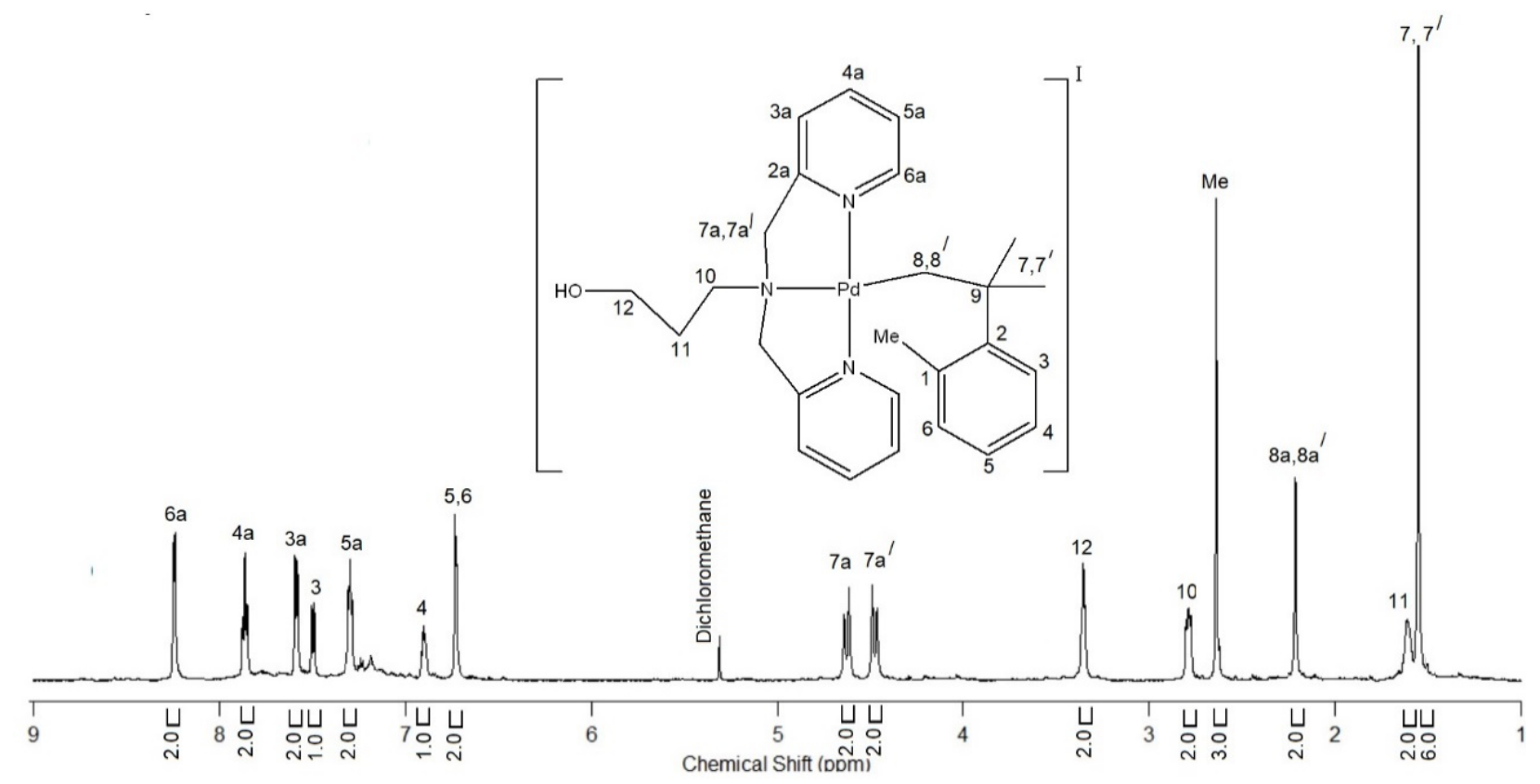

Figure S41. ${ }^{1} \mathrm{H}$ NMR spectrum of 13, $\left[\mathrm{Pd}\left(\mathrm{CH}_{2} \mathrm{CMe}_{2} \mathrm{C}_{6} \mathrm{H}_{4}-\mathrm{CH}_{3}\right)\left(\kappa^{3}-N, N^{\prime}, N^{\prime \prime}-\mathbf{L 1}\right)\right][\mathrm{I}]$, at $25^{\circ} \mathrm{C}(600$ $\mathrm{MHz}, \mathrm{CD}_{2} \mathrm{Cl}_{2}$ ).

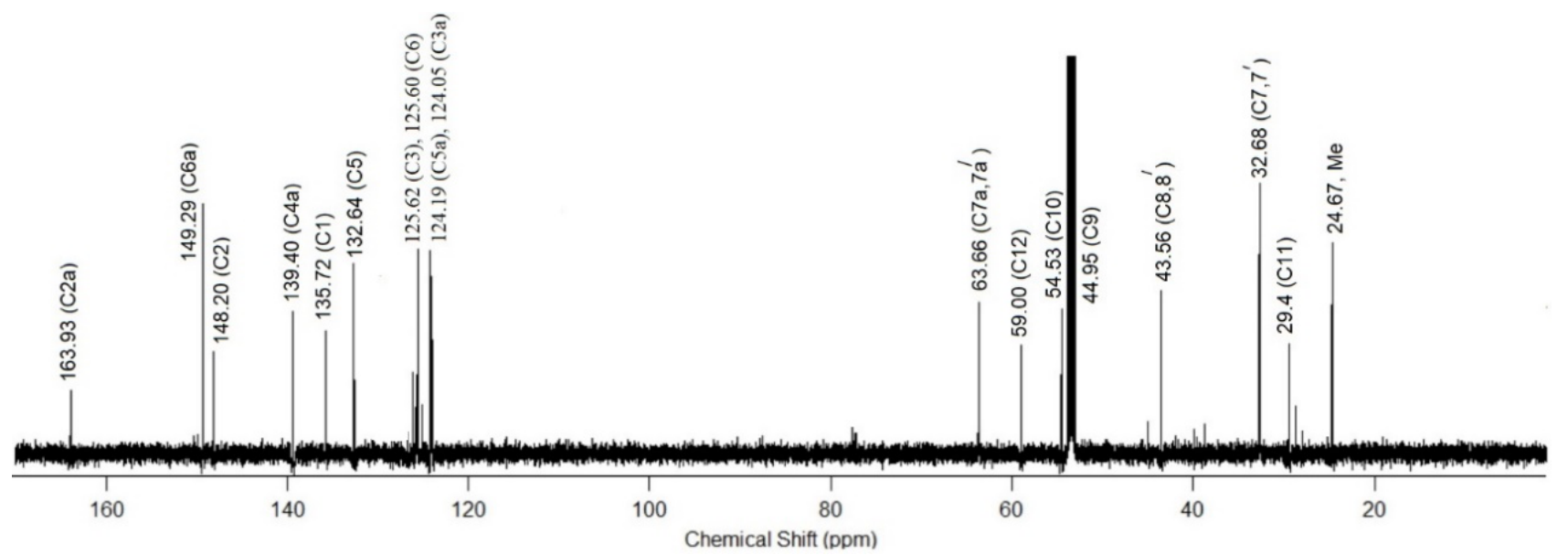

Figure S42. ${ }^{13} \mathrm{C}\left\{{ }^{1} \mathrm{H}\right\}$ NMR spectrum of 13, $\left[\mathrm{Pd}\left(\mathrm{CH}_{2} \mathrm{CMe}_{2} \mathrm{C}_{6} \mathrm{H}_{4}-\mathrm{CH}_{3}\right)\left(\kappa^{3}-N, N^{\prime}, N^{\prime \prime}-\mathbf{L} \mathbf{1}\right)\right][\mathrm{I}]$, acquired at $25^{\circ} \mathrm{C}\left(151 \mathrm{MHz}, \mathrm{CD}_{2} \mathrm{Cl}_{2}\right)$. 


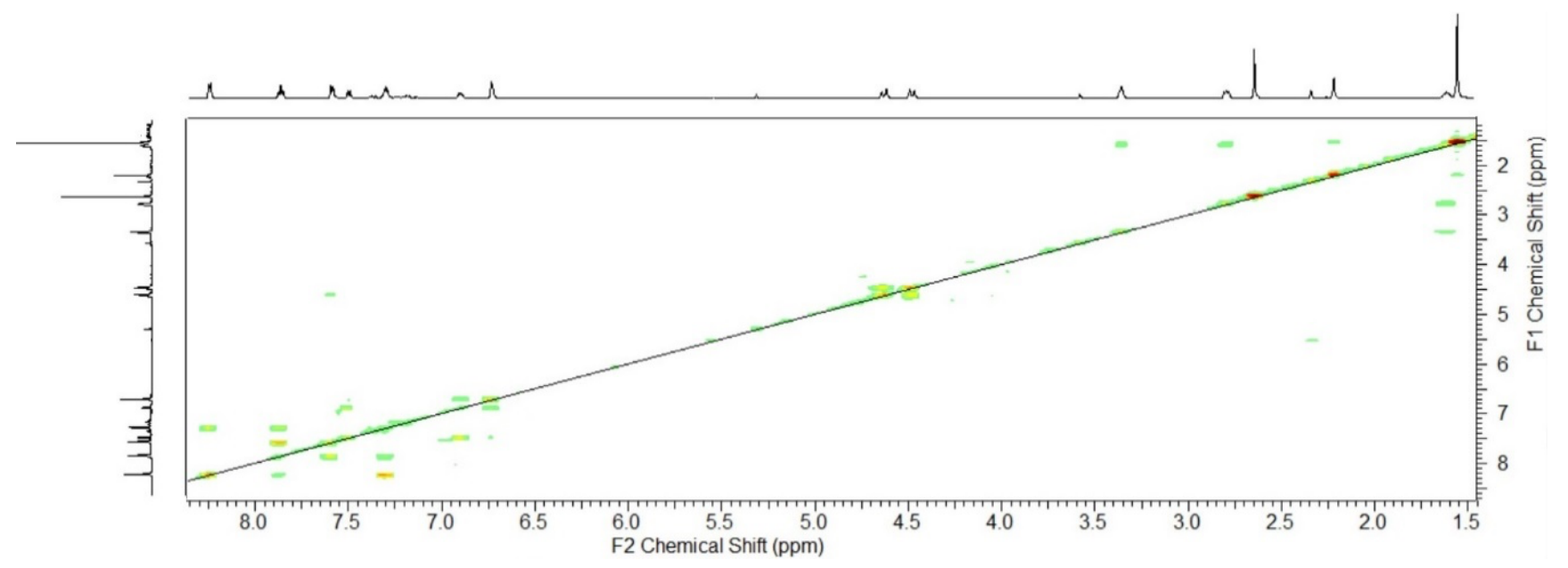

Figure S43. ${ }^{1} \mathrm{H}-{ }^{1} \mathrm{H}$ gCOSY NMR spectrum of 13, $\left[\mathrm{Pd}\left(\mathrm{CH}_{2} \mathrm{CMe}_{2} \mathrm{C}_{6} \mathrm{H}_{4}-\mathrm{CH}_{3}\right)\left(\kappa^{3}-N, N^{\prime}, N^{\prime \prime}-\mathbf{L} \mathbf{1}\right)\right][\mathrm{I}]$, at $25{ }^{\circ} \mathrm{C}\left(600 \mathrm{MHz}, \mathrm{CD}_{2} \mathrm{Cl}_{2}\right)$.

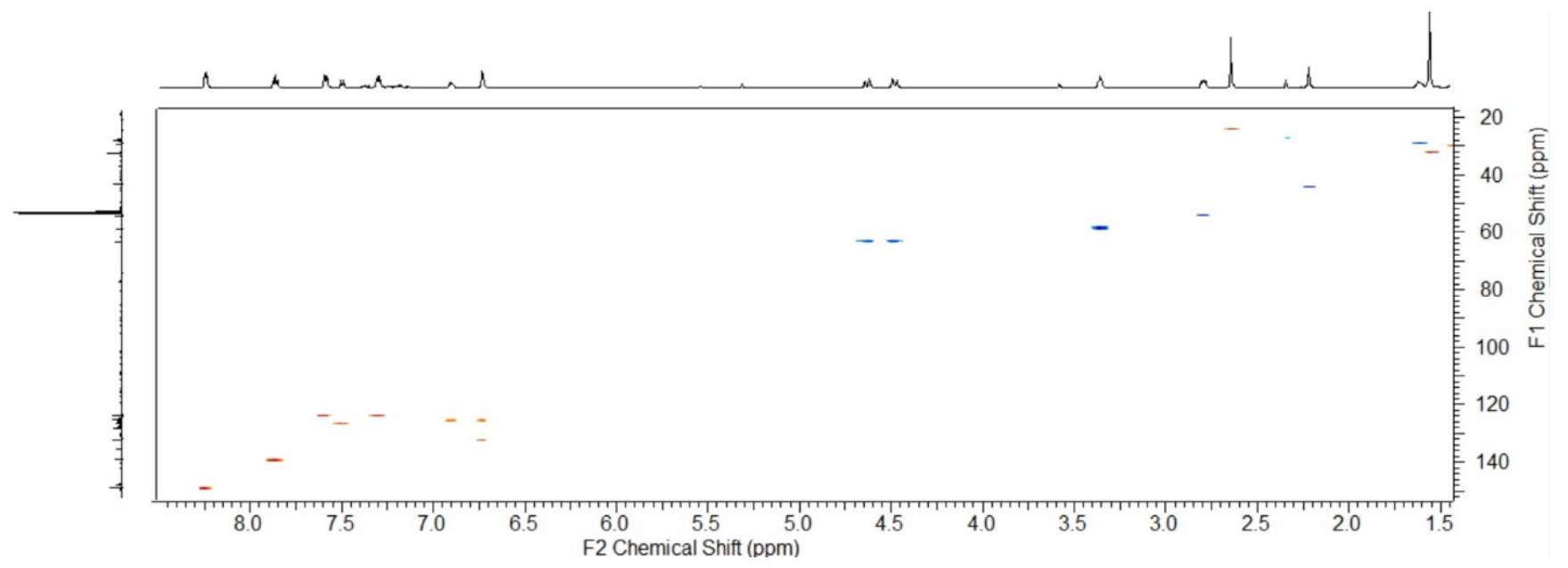

Figure S44. ${ }^{1} \mathrm{H}-{ }^{13} \mathrm{C}$ HSQC NMR spectrum of 13, $\left[\mathrm{Pd}\left(\mathrm{CH}_{2} \mathrm{CMe}_{2} \mathrm{C}_{6} \mathrm{H}_{4}-\mathrm{CH}_{3}\right)\left(\kappa^{3}-N, N^{\prime}, N^{\prime \prime}-\mathbf{L} \mathbf{1}\right)\right][\mathrm{I}]$, at $25{ }^{\circ} \mathrm{C}\left(600 \mathrm{MHz}, \mathrm{CD}_{2} \mathrm{Cl}_{2}\right)$. 


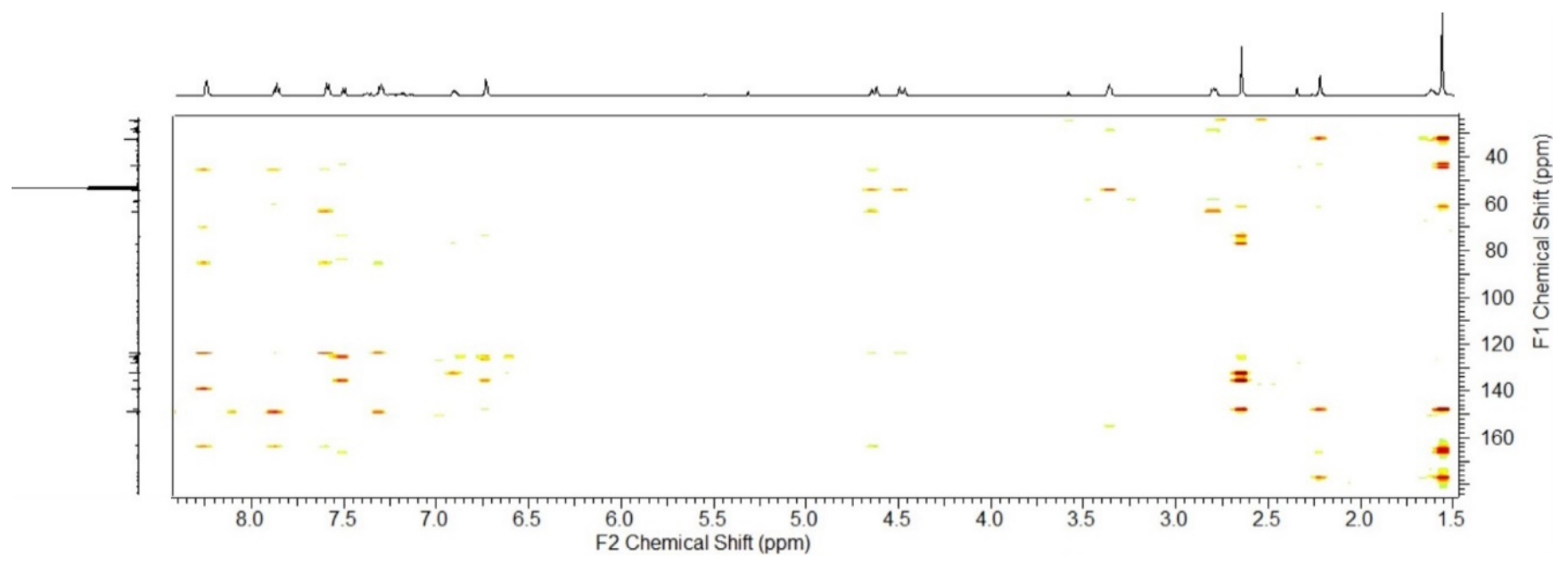

Figure S45. ${ }^{1} \mathrm{H}-{ }^{13} \mathrm{C}$ HMBC NMR spectrum of 13, $\left[\mathrm{Pd}\left(\mathrm{CH}_{2} \mathrm{CMe}_{2} \mathrm{C}_{6} \mathrm{H}_{4}-\mathrm{CH}_{3}\right)\left(\kappa^{3}-N, N^{\prime}, N^{\prime \prime}-\mathbf{L} 1\right)\right][\mathrm{I}]$, at $25^{\circ} \mathrm{C}\left(600 \mathrm{MHz}, \mathrm{CD}_{2} \mathrm{Cl}_{2}\right)$.

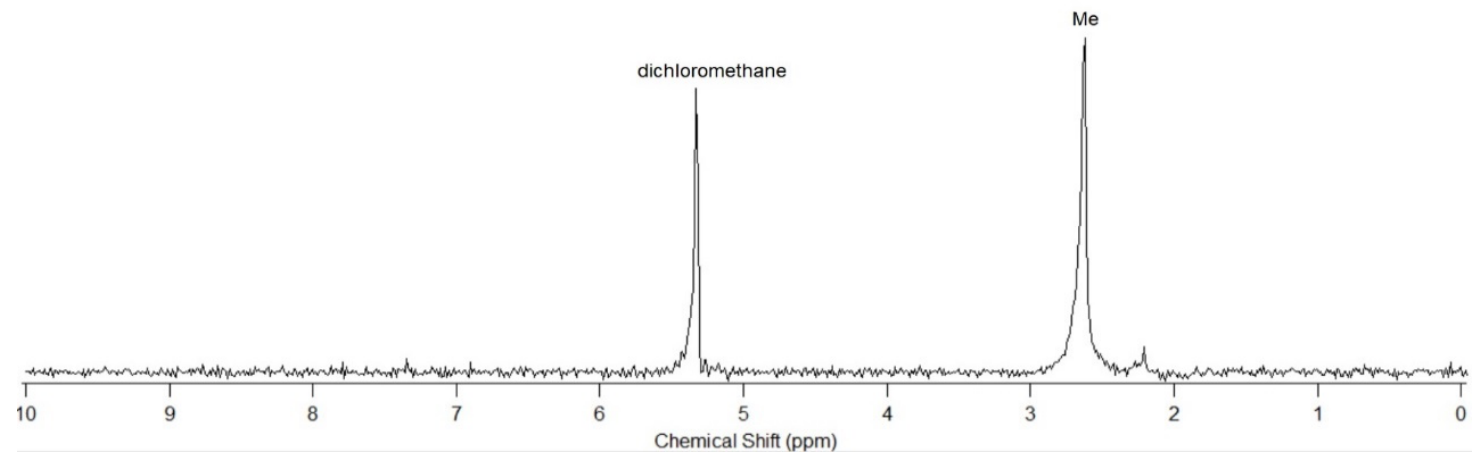

Figure S46. ${ }^{2} \mathrm{H}$ NMR spectrum of $\mathbf{1 3 -} \boldsymbol{d}_{3}$, $\left[\mathrm{Pd}\left(\mathrm{CH}_{2} \mathrm{CMe}_{2} \mathrm{C}_{6} \mathrm{H}_{4}-\mathrm{CD}_{3}\right)\left(\kappa^{3}-N, N^{\prime}, N^{\prime \prime}-\mathbf{L 1}\right)\right][\mathrm{I}]$, at $25{ }^{\circ} \mathrm{C}$ (92 MHz, $\mathrm{CH}_{2} \mathrm{Cl}_{2}$ ). 


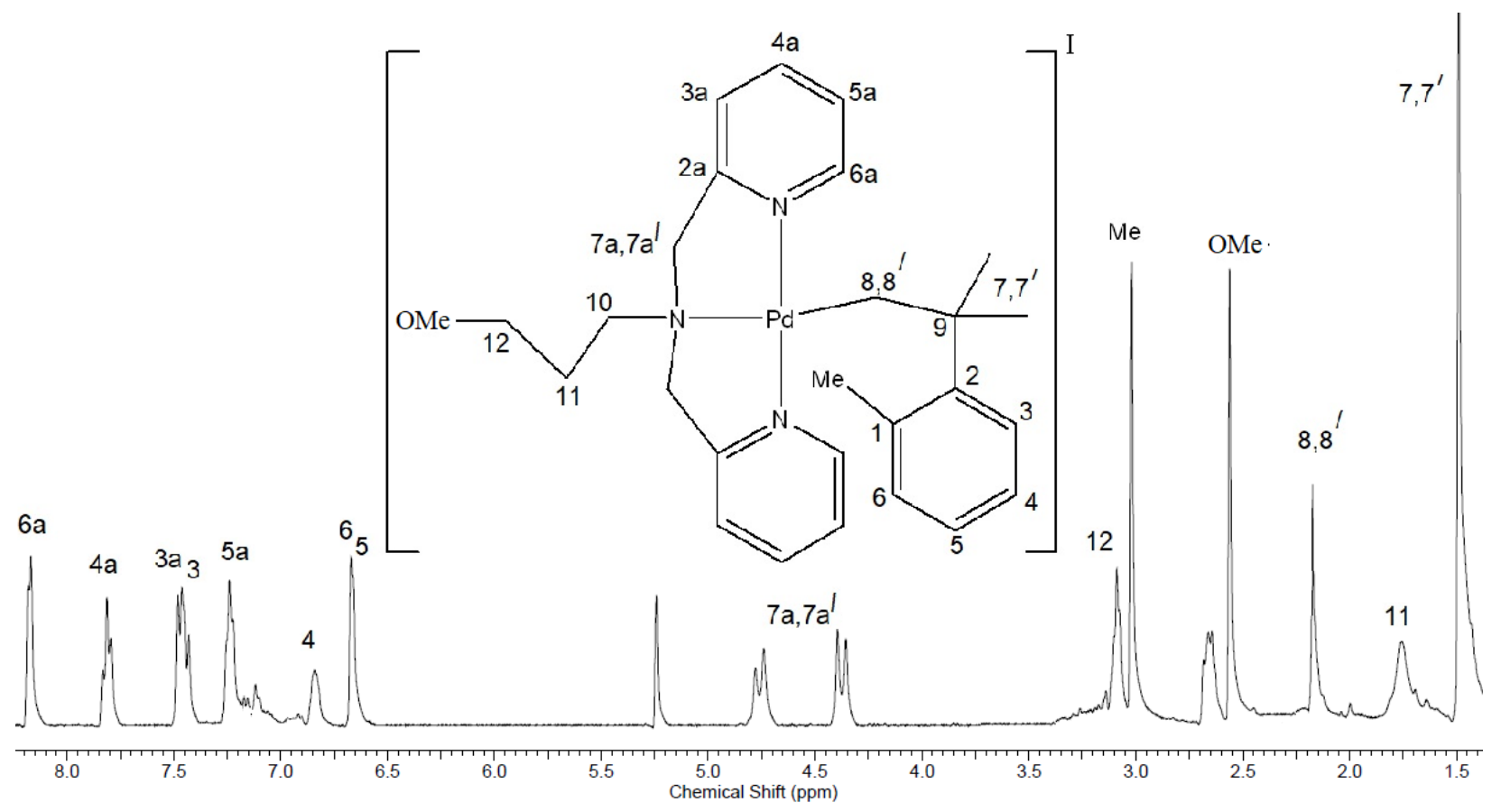

Figure S47. ${ }^{1} \mathrm{H}$ NMR spectrum of 14, $\left[\mathrm{Pd}\left(\mathrm{CH}_{2} \mathrm{CMe}_{2} \mathrm{C}_{6} \mathrm{H}_{4}-\mathrm{CH}_{3}\right)\left(\kappa^{3}-N, N^{\prime}, N^{\prime \prime}-\mathbf{L} 2\right)\right][\mathrm{I}]$, at $25^{\circ} \mathrm{C}(600$ $\left.\mathrm{MHz}, \mathrm{CD}_{2} \mathrm{Cl}_{2}\right)$.

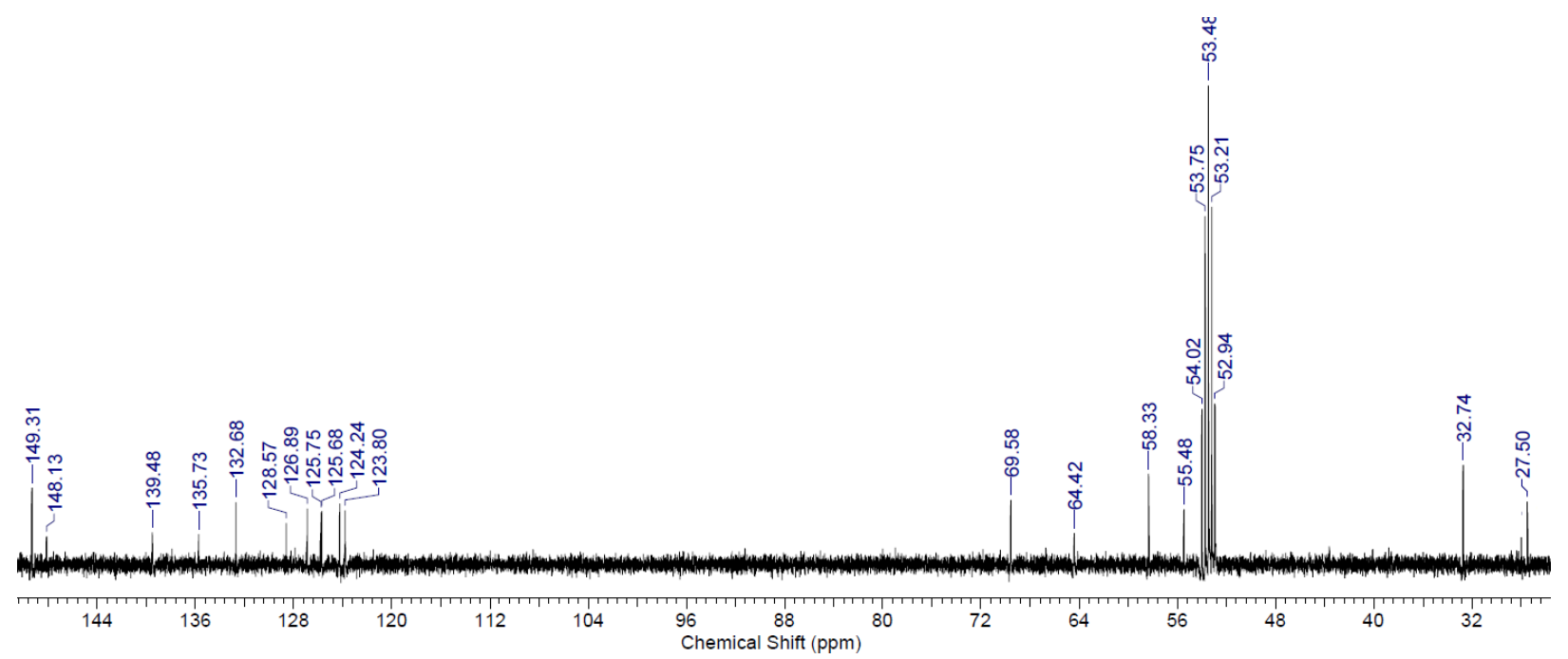

Figure S48. ${ }^{13} \mathrm{C}\left\{{ }^{1} \mathrm{H}\right\}$ NMR spectrum of 14, $\left[\mathrm{Pd}\left(\mathrm{CH}_{2} \mathrm{CMe}_{2} \mathrm{C}_{6} \mathrm{H}_{4}-\mathrm{CH}_{3}\right)\left(\kappa^{3}-N, N^{\prime}, N^{\prime \prime}-\mathrm{L2}\right)\right][\mathrm{I}]$, at 25 ${ }^{\circ} \mathrm{C}\left(151 \mathrm{MHz}, \mathrm{CD}_{2} \mathrm{Cl}_{2}\right)$. 


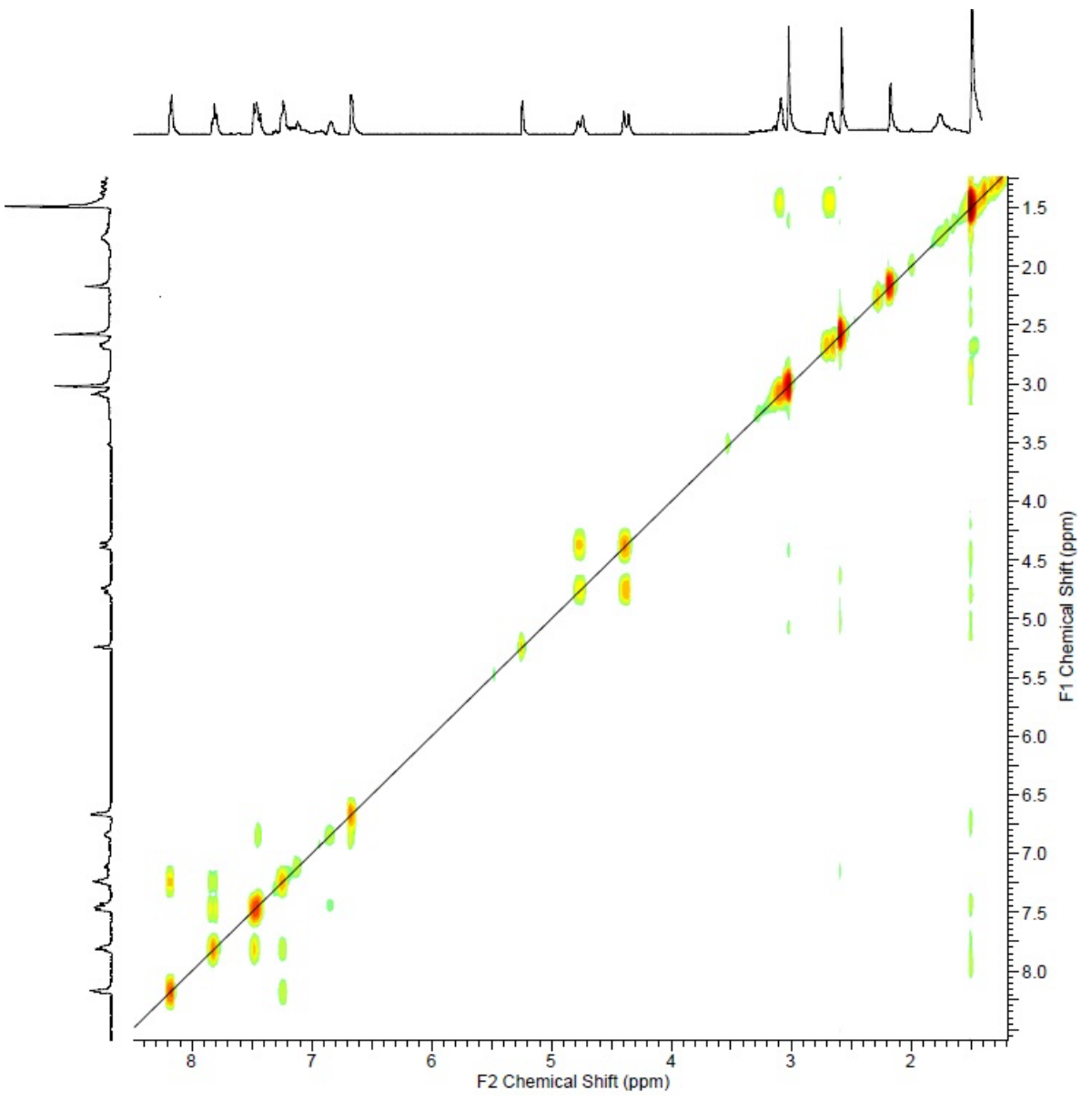

Figure S49. ${ }^{1} \mathrm{H}-{ }^{1} \mathrm{H}$ gCOSY NMR spectrum of 14, $\left[\mathrm{Pd}\left(\mathrm{CH}_{2} \mathrm{CMe}_{2} \mathrm{C}_{6} \mathrm{H}_{4}-\mathrm{CH}_{3}\right)\left(\kappa^{3}-N, N^{\prime}, N^{\prime \prime}-\mathbf{L} 2\right)\right][\mathrm{I}]$, acquired at $25^{\circ} \mathrm{C}\left(600 \mathrm{MHz}, \mathrm{CD}_{2} \mathrm{Cl}_{2}\right)$.

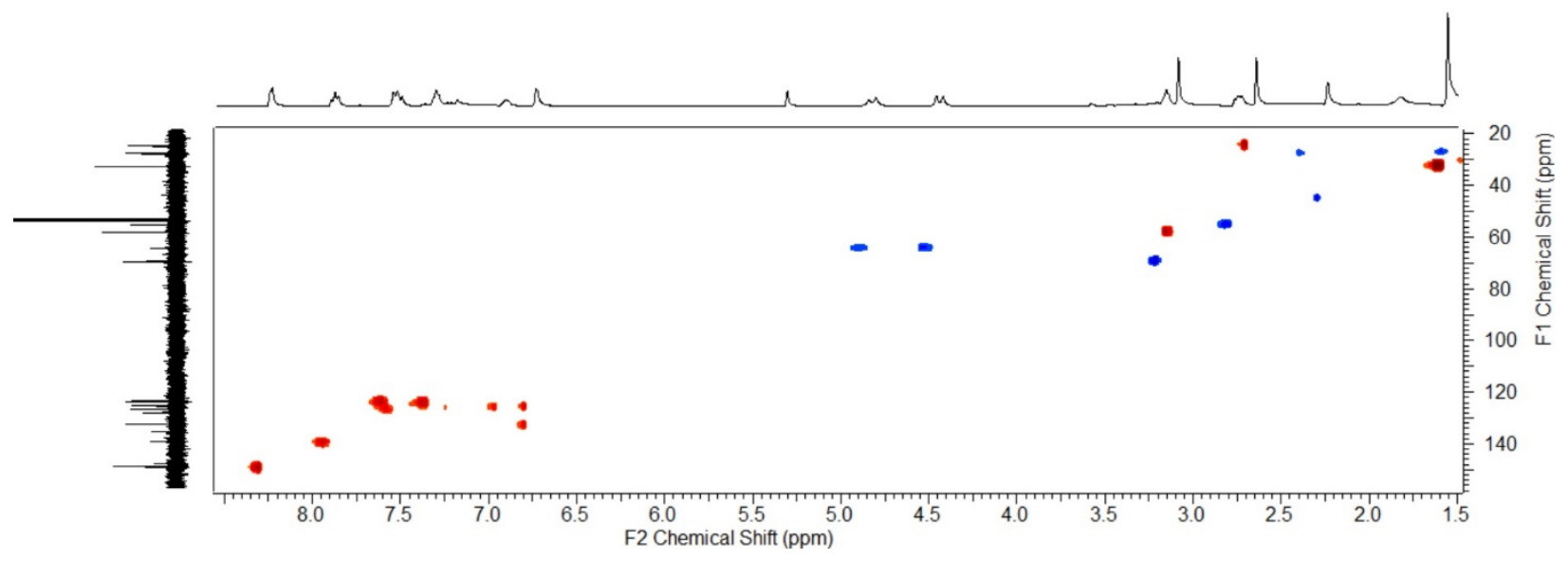

Figure S50. ${ }^{1} \mathrm{H}_{-}{ }^{13} \mathrm{C}$ HSQC NMR spectrum of 14, $\left[\mathrm{Pd}\left(\mathrm{CH}_{2} \mathrm{CMe}_{2} \mathrm{C}_{6} \mathrm{H}_{4}-\mathrm{CH}_{3}\right)\left(\kappa^{3}-N, N^{\prime}, N^{\prime \prime}-\mathbf{L} 2\right)\right][\mathrm{I}]$, acquired at $25^{\circ} \mathrm{C}\left(600 \mathrm{MHz}, \mathrm{CD}_{2} \mathrm{Cl}_{2}\right)$. 


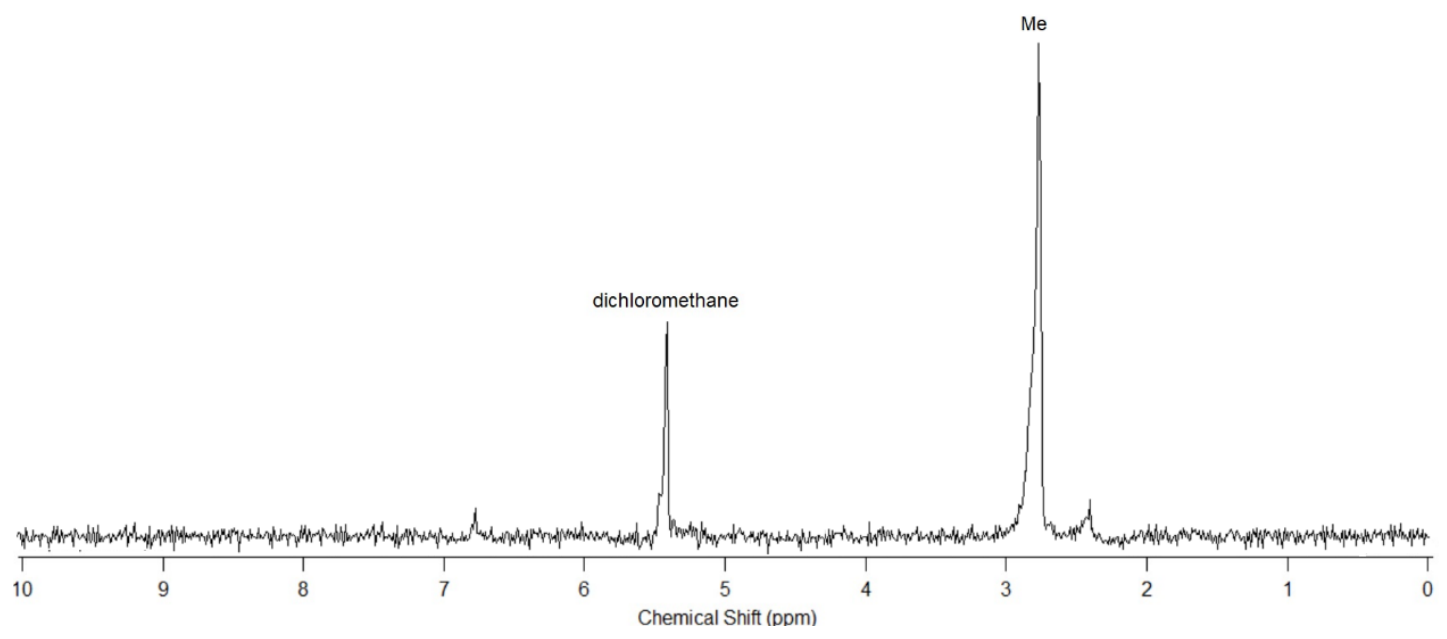

Figure S51. ${ }^{2} \mathrm{H}$ NMR spectrum of 14- $\boldsymbol{d}_{3}$, $\left[\mathrm{Pd}\left(\mathrm{CH}_{2} \mathrm{CMe}_{2} \mathrm{C}_{6} \mathrm{H}_{4}-\mathrm{CD}_{3}\right)\left(\kappa^{3}-N, N^{\prime}, N^{\prime \prime}-\mathbf{L} 2\right)\right][\mathrm{I}]$, at $25{ }^{\circ} \mathrm{C}$ (92 $\mathrm{MHz}, \mathrm{CH}_{2} \mathrm{Cl}_{2}$ ).

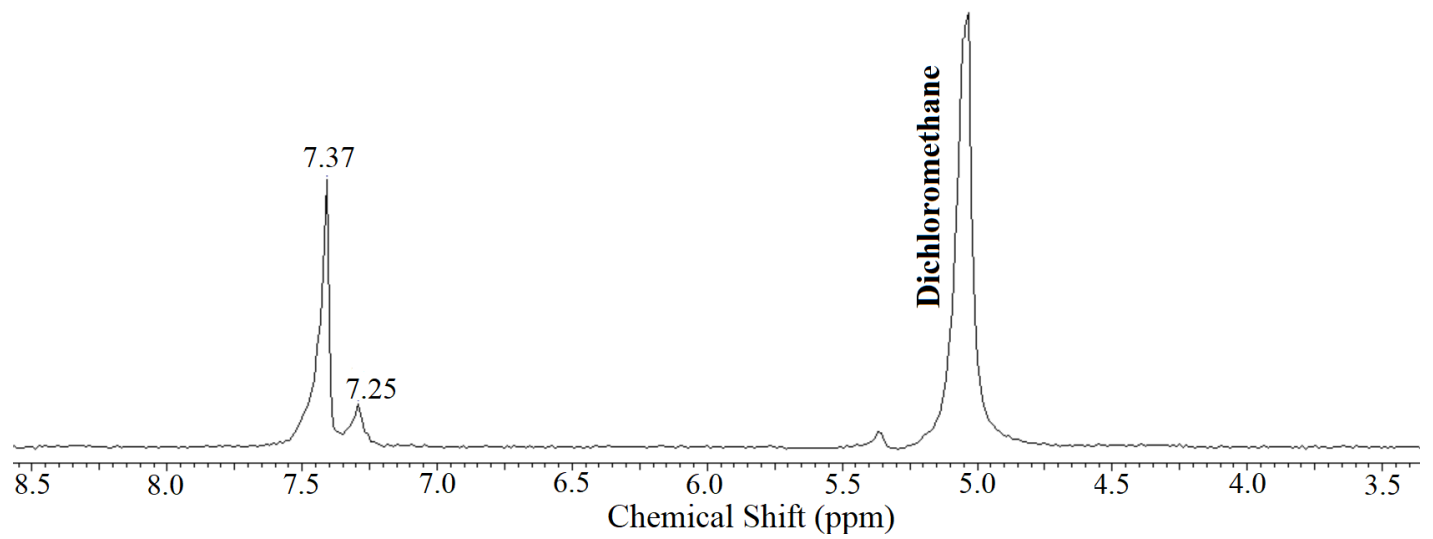

Figure S52. ${ }^{2} \mathrm{H}$ NMR spectrum after the reductive elimination from 4 at $25{ }^{\circ} \mathrm{C}(92 \mathrm{MHz}, 1: 1$ $\left.\mathrm{CH}_{2} \mathrm{Cl}_{2}: \mathrm{D}_{2} \mathrm{O}\right)$. 
III - IR Spectra

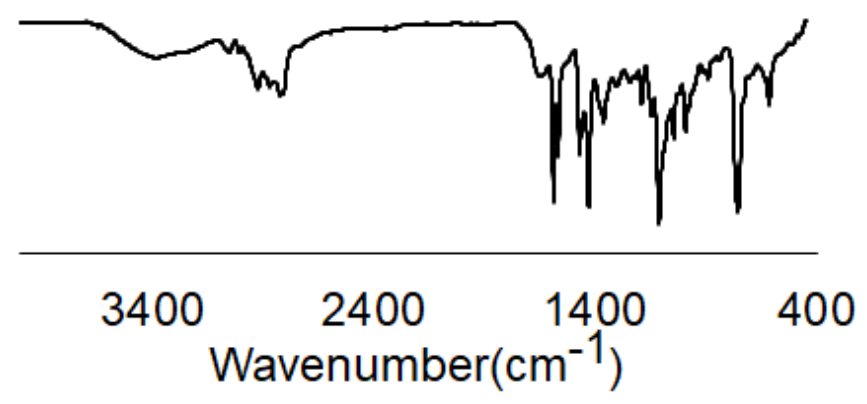

Figure S53. ATR-FTIR spectrum of solid L2

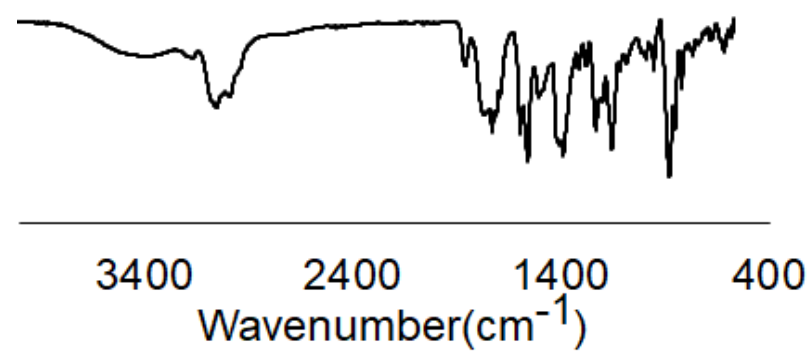

Figure S54. ATR-FTIR spectrum of solid 2, $\left[\mathrm{Pd}\left(\mathrm{CH}_{2} \mathrm{CMe}_{2} \mathrm{C}_{6} \mathrm{H}_{4}\right)\left(\kappa^{2}-N, N^{\prime}-\mathbf{L 2}\right)\right]$

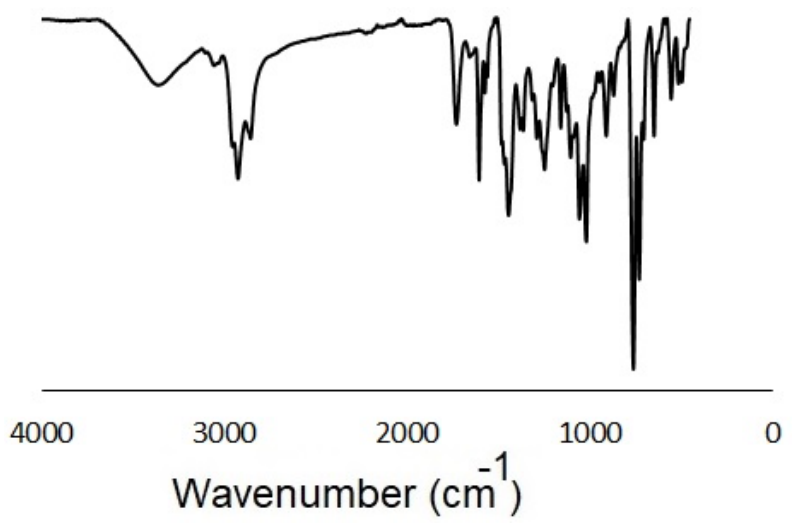

Figure S55. ATR-FTIR spectrum of solid 3, $\left[\mathrm{PdI}\left(\mathrm{CH}_{2} \mathrm{CMe}_{2} \mathrm{C}_{6} \mathrm{H}_{4}\right)\left(\kappa^{3}-N, N^{\prime}, N^{\prime \prime}-\mathbf{L 1}\right)\right][\mathrm{I}]$. 


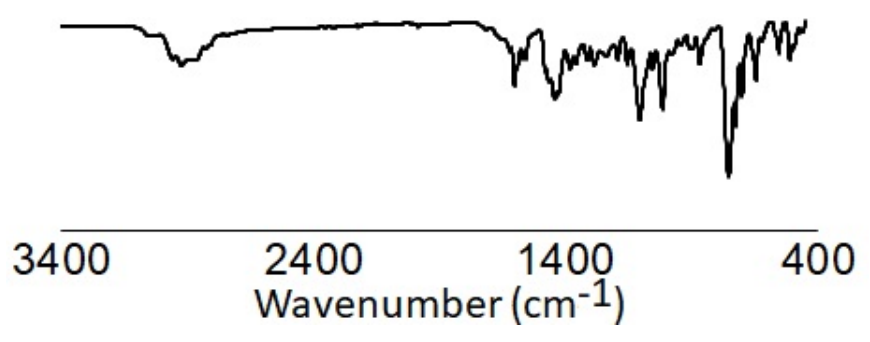

Figure S56. ATR-FTIR spectrum of solid 4, $\left[\mathrm{PdI}\left(\mathrm{CH}_{2} \mathrm{CMe}_{2} \mathrm{C}_{6} \mathrm{H}_{4}\right)\left(\kappa^{3}-N, N^{\prime}, N^{\prime \prime}-\mathbf{L 2}\right)\right][\mathrm{I}]$.

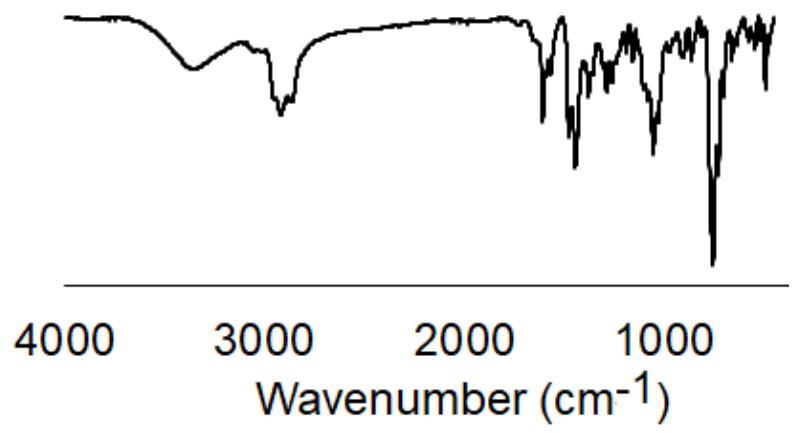

Figure S57. ATR-FTIR spectrum of solid 5, $\left[\mathrm{PdBr}\left(\mathrm{CH}_{2} \mathrm{CMe}_{2} \mathrm{C}_{6} \mathrm{H}_{4}\right)\left(\kappa^{3}-N, N^{\prime}, N^{\prime \prime}-\mathbf{L 1}\right)\right][\mathrm{Br}]$.

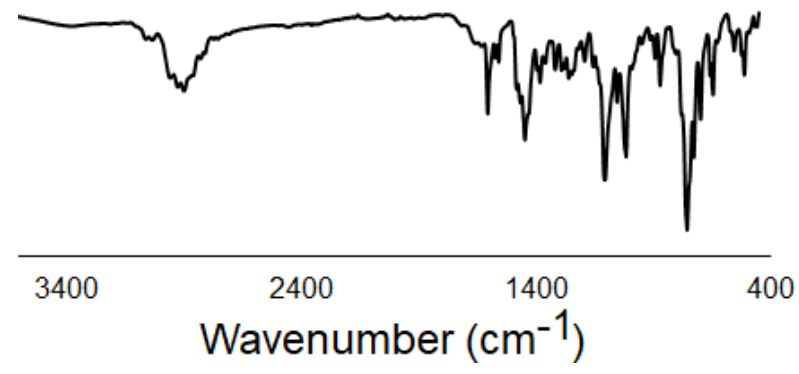

Figure S58. ATR-FTIR spectrum of solid 6, $\left[\mathrm{PdBr}\left(\mathrm{CH}_{2} \mathrm{CMe}_{2} \mathrm{C}_{6} \mathrm{H}_{4}\right)\left(\kappa^{3}-N, N^{\prime}, N^{\prime \prime}-\mathbf{L 2}\right)\right][\mathrm{Br}]$.

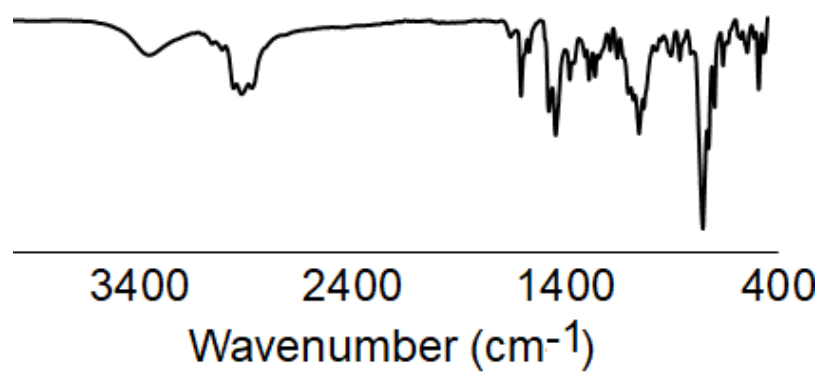

Figure S59. ATR-FTIR spectrum of solid 7, $\left[\operatorname{Pd}\left(\kappa^{3}-N, N^{\prime}, N^{\prime \prime}-\mathbf{L} \mathbf{1}\right)\right][\mathrm{I}]$. 


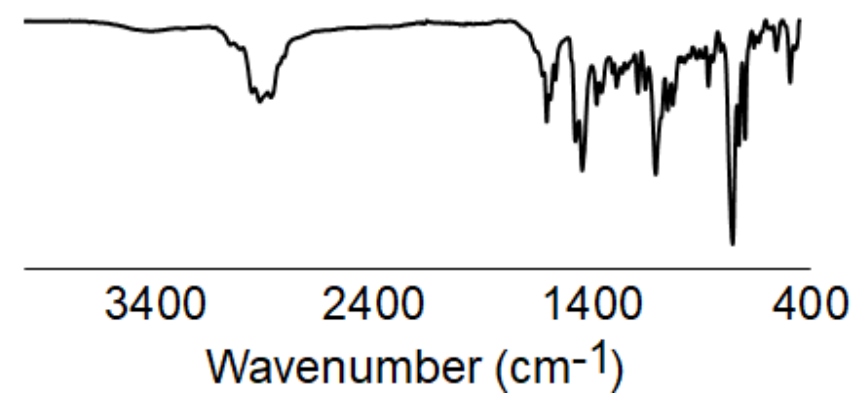

Figure S60. ATR-FTIR spectrum of solid 8, $\left[\operatorname{Pd}\left(\kappa^{3}-N, N^{\prime}, N^{\prime \prime}-\mathbf{L} 2\right)\right][\mathrm{I}]$.

\section{IV - UV-vis Spectroscopy Kinetic Data}
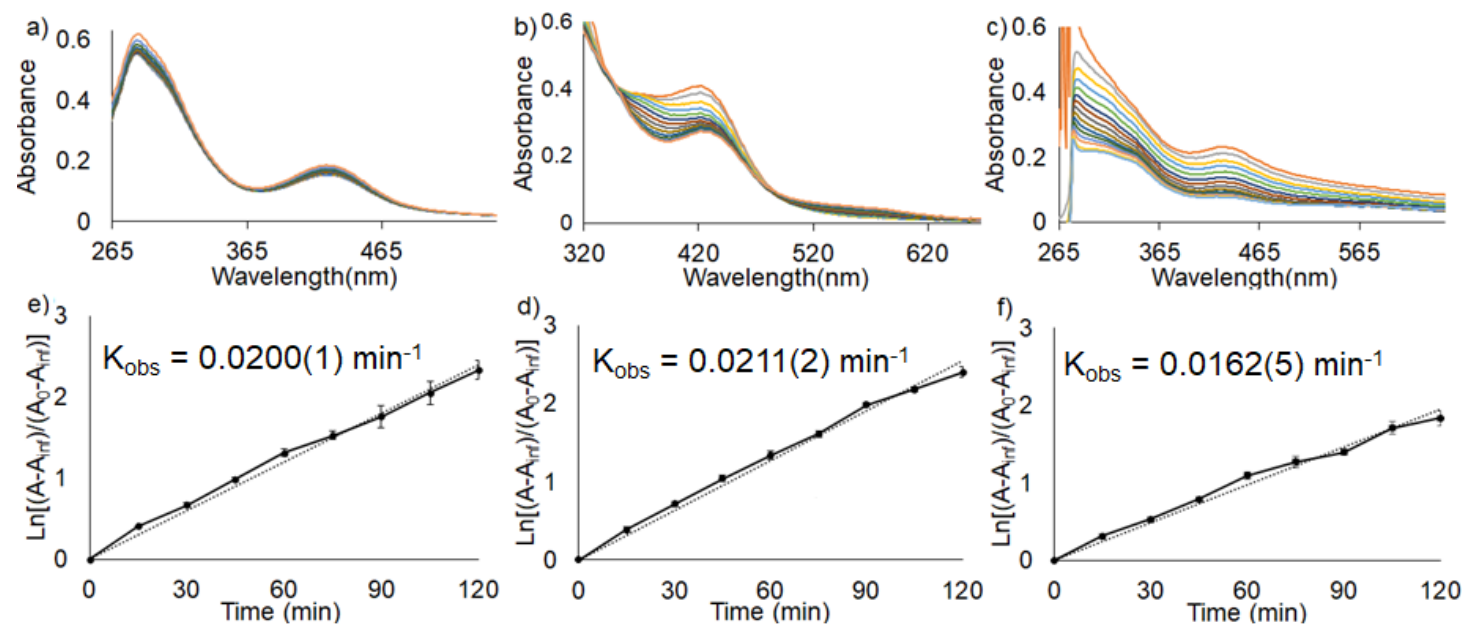

Figure S61. UV-visible absorption spectra acquired over time (top) of complex $\mathbf{3}\left(8.33 \times 10^{-3} \mathrm{M}\right)$ undergoing reductive elimination at $50{ }^{\circ} \mathrm{C}$ in: a) methanol; b) chloroform; and c) benzene. The corresponding first order kinetic plots (bottom) of $\ln \left[\mathrm{A}-\mathrm{A}_{\infty}\right] /\left[\mathrm{A}_{0}-\mathrm{A}_{\infty}\right]$ versus time in e) methanol d) chloroform f) benzene.
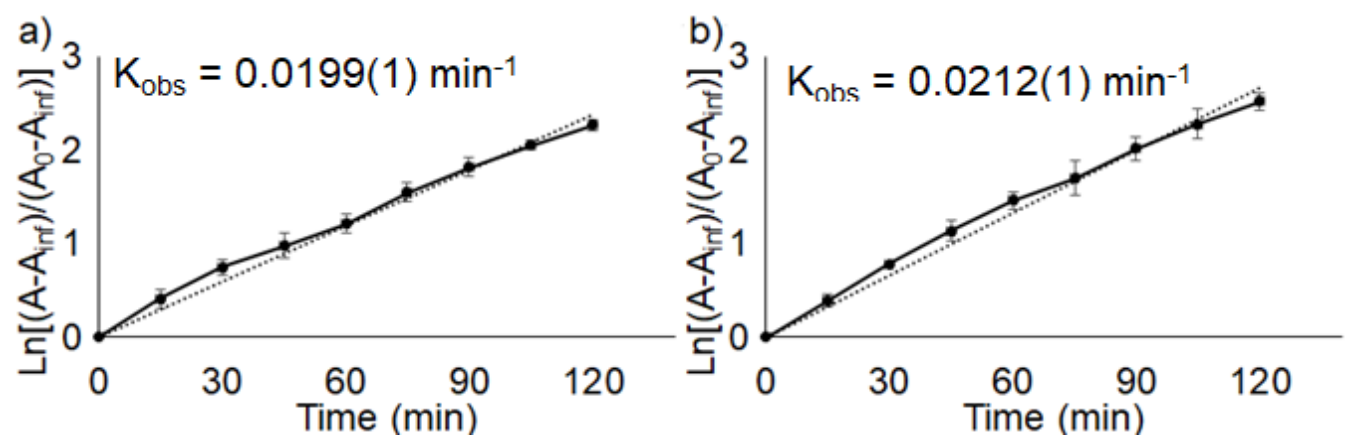

Figure S62. First order plot of $\ln \left[\mathrm{A}-\mathrm{A}_{\infty}\right] /\left[\mathrm{A}_{0}-\mathrm{A}_{\infty}\right]$ versus time for 3 in methanol $\left(8.33 \times 10^{-3} \mathrm{M}\right)$ in the presence of excess a) sodium iodide b) pyridine. 

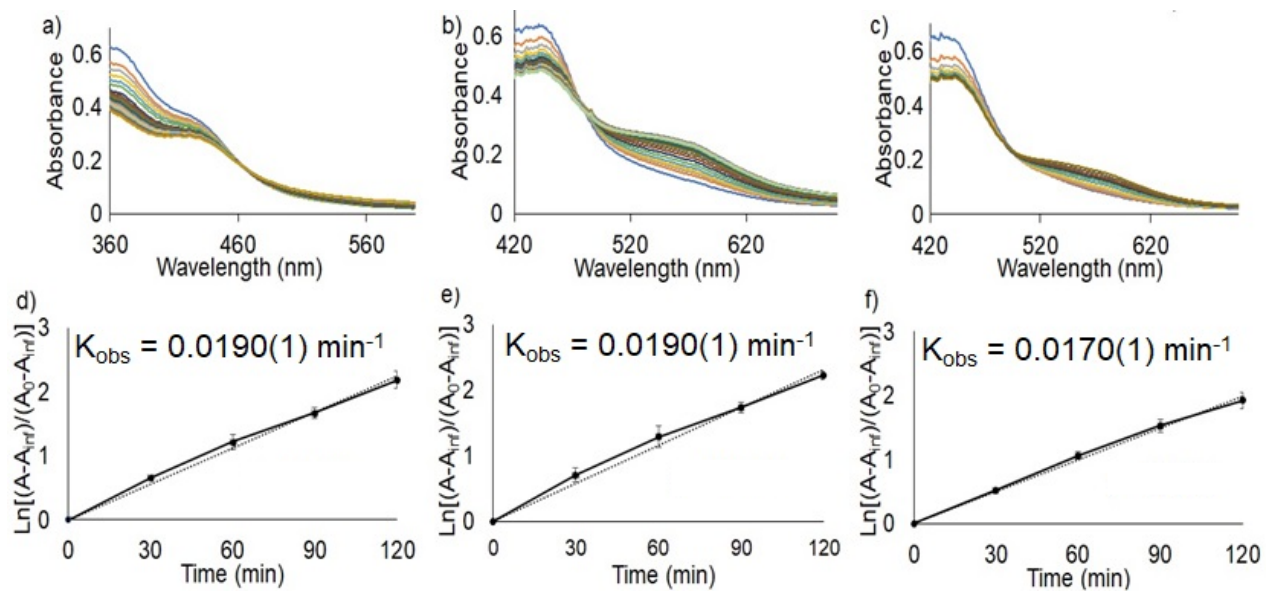

Figure S63. UV-visible absorption spectra acquired over time (top) of complex $4\left(8.33 \times 10^{-3} \mathrm{M}\right)$ undergoing reductive elimination at $50{ }^{\circ} \mathrm{C}$ in a) methanol b) chloroform; and c) benzene $(1.30 \times$ $10^{-3} \mathrm{M}$ ). The corresponding first order kinetic plots (bottom) of $\ln \left[\mathrm{A}-\mathrm{A}_{\infty}\right] /\left[\mathrm{A}_{0}-\mathrm{A}_{\infty}\right]$ versus time in e) methanol d) chloroform f) benzene.
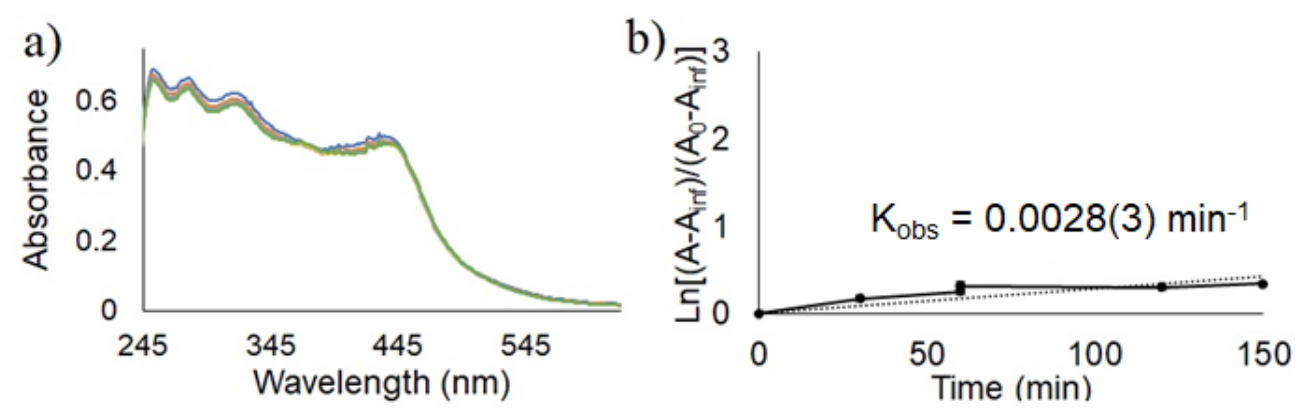

Figure S64. a) UV-visible absorption spectra of $4 a$ in chloroform $\left(4.33 \times 10^{-3} \mathrm{M}\right)$ and b) corresponding first order plot of $\ln \left[\mathrm{A}-\mathrm{A}_{\infty}\right] /\left[\mathrm{A}_{0}-\mathrm{A}_{\infty}\right]$ versus time.
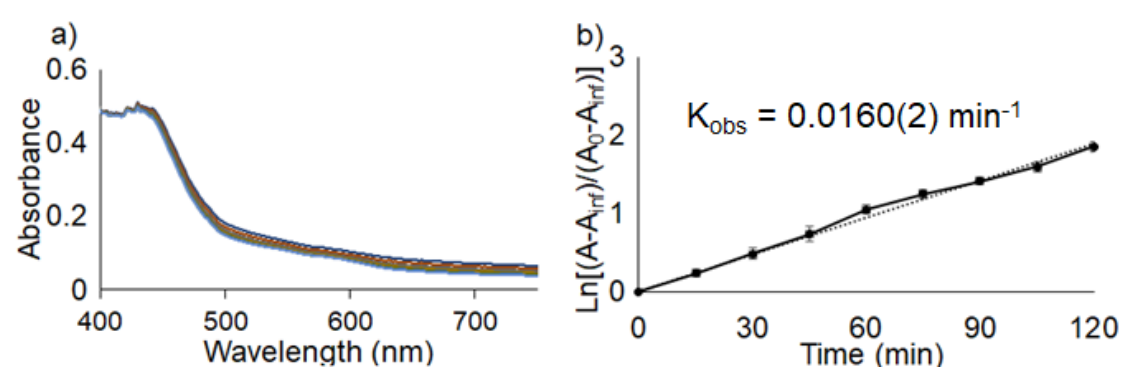

Figure S65. a) UV-visible absorption spectra of 5 in chloroform $\left(8.33 \times 10^{-3} \mathrm{M}\right)$ and b) corresponding first order plot of $\ln \left[\mathrm{A}-\mathrm{A}_{\infty}\right] /\left[\mathrm{A}_{0}-\mathrm{A}_{\infty}\right]$ versus time. 


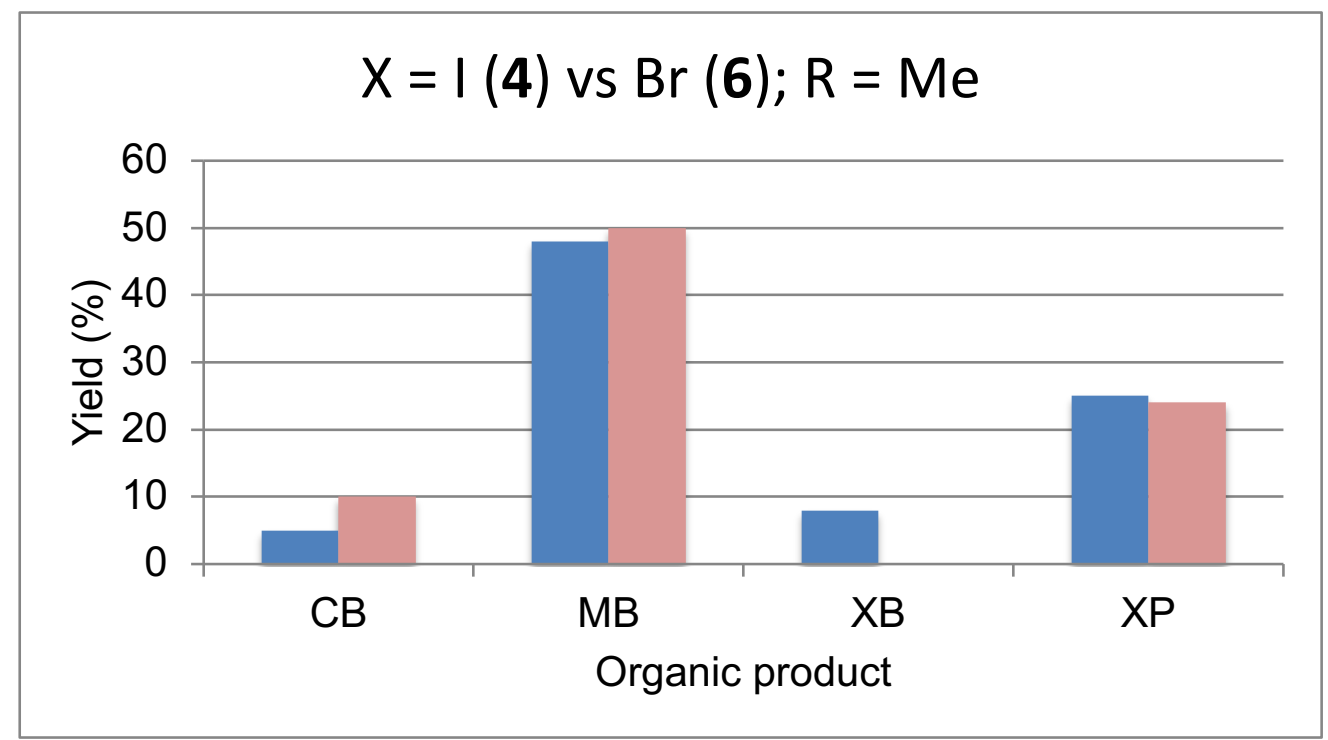

Figure S66. Observed organic products after reductive elimination from L2 complexes 4 (blue) and 6 (red) in $\mathrm{CDCl}_{3}$ solution.

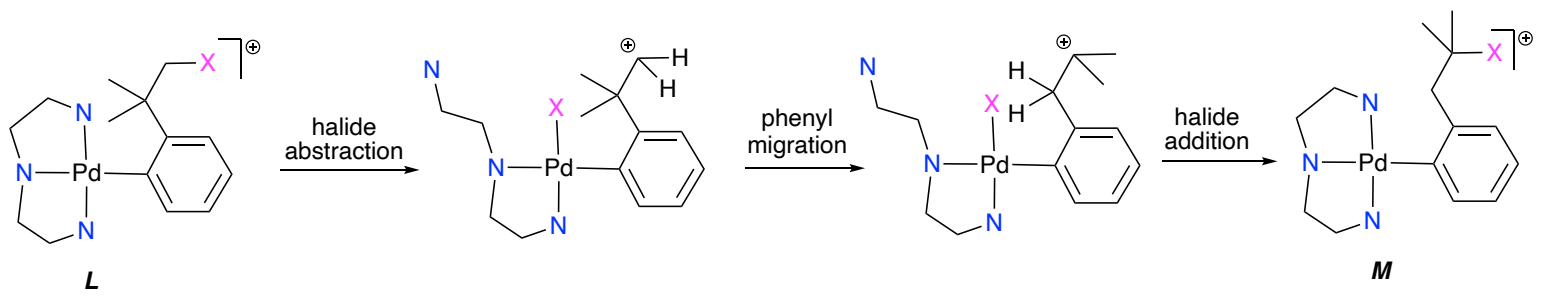

Scheme S1. Proposed sequence for the neophyl phenyl migration from intermediates $\boldsymbol{L}$ to $\boldsymbol{M}$. $N, N^{\prime}, N^{\prime \prime}$-ligand $=\mathbf{L} \mathbf{1}$ or $\mathbf{L} \mathbf{2}$. 


\section{V - Crystallographic Details}

Data Collection and Processing. The sample was mounted on a Mitegen polyimide micromount with a small amount of Paratone $\mathrm{N}$ oil. All X-ray measurements were made on a Bruker Kappa Axis Apex 2 diffractometer at a temperature of $110 \mathrm{~K}$. The frame integration was performed using SAINT. ${ }^{1}$ The resulting raw data was scaled and absorption corrected using a multi-scan averaging of symmetry equivalent data using SADABS. ${ }^{2}$

Structure Solution and Refinement. The structures were solved by using a dual space methodology using the SHELXT program. ${ }^{3}$ All non-hydrogen atoms were obtained from the initial solution. The hydrogen atoms were introduced at idealized positions and were allowed to ride on the parent atom. The structural model was fit to the data using full matrix least-squares based on $F^{2}$. The calculated structure factors included corrections for anomalous dispersion from the usual tabulation. The structure was refined using the SHELXL program from the SHELXTL suite of crystallographic software. ${ }^{4}$ Graphic plots were produced using the Mercury program suite. ${ }^{5}$ 
Table S1. Summary of Crystal Data for 3, 5, 7-10

\begin{tabular}{|c|c|c|c|c|c|}
\hline $\begin{array}{l}\text { Compound \# } \\
\text { (CCDC) }\end{array}$ & $\begin{array}{l}3 \text { \& } 7 \\
(2031186)\end{array}$ & $\begin{array}{l}\mathbf{5} \\
(2031187)\end{array}$ & $\begin{array}{l}\mathbf{8} \\
(2031188)\end{array}$ & $\begin{array}{l}9 \\
(2031189)\end{array}$ & $\begin{array}{l}\mathbf{1 0} \\
(2031190)\end{array}$ \\
\hline Formula & $\begin{array}{l}\mathrm{C}_{43} \mathrm{H}_{56} \mathrm{Br}_{5} \mathrm{~N}_{6} \mathrm{O}_{3} \mathrm{Pd}_{2} \\
-\left(\mathrm{CH}_{3}\right)_{2} \mathrm{CO}\end{array}$ & $\begin{array}{l}\mathrm{C}_{28} \mathrm{H}_{37} \mathrm{Br}_{2} \mathrm{~N}_{3} \mathrm{O}_{2} \mathrm{Pd} \\
\cdot\left(\mathrm{CH}_{3}\right)_{2} \mathrm{CO}\end{array}$ & $\mathrm{C}_{17} \mathrm{H}_{23} \mathrm{Cl}_{2} \mathrm{I}_{2} \mathrm{~N}_{3} \mathrm{OPd}$ & $\mathrm{C}_{15} \mathrm{H}_{19} \mathrm{Br}_{2} \mathrm{~N}_{3} \mathrm{OPd}$ & $\begin{array}{l}\mathrm{C}_{16} \mathrm{H}_{21} \mathrm{Br}_{2} \mathrm{~N}_{3} \mathrm{OPd} \\
\cdot \mathrm{H}_{2} \mathrm{O}\end{array}$ \\
\hline $\begin{array}{l}\text { Formula } \\
\text { Weight }(\mathrm{g} / \mathrm{mol})\end{array}$ & 1552.32 & 771.9 & 716.48 & 523.57 & 555.61 \\
\hline Crystal System & Triclinic & Orthorhombic & Orthorhombic & Monoclinic & Monoclinic \\
\hline Space Group & $P-1$ & $\mathrm{Pca}_{1}$ & Pbca & $P 2_{1} / \mathrm{c}$ & $P 2{ }_{1} / \mathrm{c}$ \\
\hline$a, \AA$ & $9.125(3)$ & $18.365(4)$ & $11.291(11)$ & $18.484(5)$ & $8.8407(12)$ \\
\hline$b, \AA$ & $16.078(6)$ & $8.298(2)$ & $15.345(11)$ & $9.302(4)$ & $16.336(2)$ \\
\hline$c, \AA$ & $17.370(7)$ & $38.438(10)$ & $26.52(2)$ & $21.445(8)$ & $26.695(3)$ \\
\hline$\alpha,^{\circ}$ & $74.749(13)$ & 90 & 90 & 90 & 90 \\
\hline$\beta,^{\circ}$ & $80.804(11)$ & 90 & 90 & $112.647(8)$ & $91.781(2)$ \\
\hline$\gamma,{ }^{\circ}$ & $84.646(17)$ & 90 & 90 & 90 & 90 \\
\hline $\mathrm{V}, \AA^{3}$ & $2423.9(16)$ & $5858(2)$ & $4595(7)$ & $3403(2)$ & $3853.5(9)$ \\
\hline $\begin{array}{l}\text { Min and Max } \\
2 \theta \text { for cell } \\
\text { determination, }\end{array}$ & $1.227,24.749$ & $2.455,24.864$ & $3.80,35.98$ & $2.494,24.750$ & $3.172,58.964$ \\
\hline Z & 4 & 8 & 8 & 8 & 8 \\
\hline $\mathrm{F}(000)$ & 1368 & 2864 & 2720 & 2032 & 2136 \\
\hline$\rho(\mathrm{g} / \mathrm{cm})$ & 1.953 & 1.619 & 2.071 & 2.044 & 1.884 \\
\hline$\lambda, \AA,(\mathrm{MoK} \alpha)$ & 0.71073 & 0.71073 & 0.71073 & 0.71073 & 1.54178 \\
\hline $\begin{array}{l}\text { Number of } \\
\text { reflections } \\
\text { measured }\end{array}$ & 41867 & 100603 & 36363 & 54489 & 22008 \\
\hline $\begin{array}{l}\text { Unique } \\
\text { reflections } \\
\text { measured }\end{array}$ & 8302 & 10094 & 10622 & 5814 & 5511 \\
\hline $\mathrm{R}_{1}$ & 0.0335 & 0.0513 & 0.0313 & 0.0458 & 0.0389 \\
\hline$w_{2}$ & 0.0885 & 0.1001 & 0.0636 & 0.1232 & 0.0905 \\
\hline $\mathrm{R}_{1}$ (all data) & 0.0386 & 0.0955 & 1.086 & 1.055 & 1.083 \\
\hline$w_{2}$ (all data) & 0.0967 & 0.1162 & 0.0666 & 0.1285 & 0.0919 \\
\hline GOF & 1.112 & 1.027 & 1.086 & 1.055 & 1.083 \\
\hline $\begin{array}{l}\text { Maximum } \\
\text { shift/error }\end{array}$ & 0.0001 & 0.001 & 0.003 & 0.001 & 0.002 \\
\hline
\end{tabular}




\begin{tabular}{|l|l|l|l|l|l|}
\hline $\begin{array}{l}\text { Min \& Max } \\
\text { peak heights on } \\
\text { final } \Delta \text { F Map } \\
\left(e^{-/} / \AA\right)\end{array}$ & $-1.253,3.080$ & $-1.128,1.075$ & $-1.066,1.926$ & $-1.128,1.075$ & $-0.711,4.444$ \\
\hline
\end{tabular}

Where:

$\mathrm{R}_{1}=\Sigma\left(\left|\mathrm{F}_{\mathrm{o}}\right|-\left|\mathrm{F}_{\mathrm{c}}\right|\right) / \Sigma \mathrm{F}_{\mathrm{o}}$

$\mathrm{wR}_{2}=\left[\Sigma\left(w\left(\mathrm{~F}_{\mathrm{o}}^{2}-\mathrm{F}_{\mathrm{c}}^{2}\right)^{2}\right) / \Sigma\left(w \mathrm{~F}_{\mathrm{o}}^{4}\right)\right]^{1 / 2}$

$\mathrm{GOF}=\left[\Sigma\left(w\left(\mathrm{~F}_{\mathrm{o}}^{2}-\mathrm{F}_{\mathrm{c}}^{2}\right)^{2}\right) /(\text { No. of reflns. - No. of params. })\right]^{1 / 2}$

\section{References}

1. Bruker-Nonius; SAINT; version; 2013.8, 2013, Bruker-Nonius, Madison, WI 53711, USA

2. Bruker-Nonius; SADABS; version; 2012.1, 2012, Bruker-Nonius, Madison, WI 53711, USA.

3. Sheldrick, G., SHELXT - Integrated space-group and crystal-structure determination. Acta Crystallogr. Sect. A 2015, A71 (1), 3-8.

4. Sheldrick, G., Crystal structure refinement with SHELXL. Acta Crystallogr. Sect. C 2015, C71 (1), 3-8.

5. Macrae, C. F.; Bruno, I. J.; Chisholm, J. A.; Edgington, P. R.; McCabe, P.; Pidcock, E.; Rodriguez-Monge, L.; Taylor, R.; van de Streek, J.; Wood, P. A., Mercury CSD 2.0 - new features for the visualization and investigation of crystal structures. J. Appl. Cryst. 2008, 41 (2), 466-470. 